\title{
Parameter identification methods for visco- and hyperelastic material models
}

\author{
Michael A. Kraus $($ Miriam Schuster $\mathbb{D}$ - \\ Johannes Kuntsche $\mathbb{D}$ - Geralt Siebert • \\ Jens Schneider(iD
}

Received: 17 March 2017 / Accepted: 22 May 2017 / Published online: 7 July 2017

(C) Springer International Publishing Switzerland 2017

\begin{abstract}
Many materials in modern civil engineering applications, such as interlayers for laminated safety glass, are polymer-based. These materials are showing distinct viscoelastic (strain-rate) and temperature dependent behaviour. In literature, different mathematical representations of these phenomena exist. A common one is the 'Prony-series' representation, which is implemented in many state-of-the-art Finite-ElementAnalysis-Software to incorporate linear viscoelastic material behaviour. The Prony-parameters at a certain reference temperature can either be determined by relaxation or retardation experiments in the time domain or with a steady state oscillation in the frequency domain in the so called 'Dynamic Mechanical
\end{abstract}

\footnotetext{
M. A. Kraus ( $\square)$

Institute and Laboratory for Structural Engineering,

University of German Armed Forces Munich, Neubiberg,

Germany

e-mail:m.kraus@unibw.de

M. Schuster $(\varangle)$

Institute of Structural Mechanics and Design, Technische

Universität Darmstadt, Darmstadt, Germany

e-mail: schuster@ismd.tu-darmstadt.de

J. Kuntsche

Ingenieurbüro für Tragwerksplanung,

ProfessorPfeiferandPartner, Darmstadt, Germany

G. Siebert

Structural Design and Building Physics, University of

German Armed Forces Munich, Neubiberg, Germany

J. Schneider

Structural Mechanics and Design, Technische Universität Darmstadt, Darmstadt, Germany
}

Thermal Analysis' followed by a 'Time-TemperatureSuperposition-Principle'. However, present research shows that polymeric materials also may need to have constitutive equations which include hyperelasticity (nonlinear stress-strain behaviour in a quasi-static condition) when undergoing large deformations, so that the material model should be expanded for a more realistic representation in numerical simulations. A novel method for the whole identification process for a numerical material model in terms of a linear Generalized Maxwell Model (Prony-series) based on experimental data will be presented. Furthermore, material parameters for different hyperelastic material models based on experimental investigations will be shown and compared. Future research activities as well as extensions of the presented novel method are also highlighted within this paper.

Keywords Laminated safety glass - Viscoelasticity · DMTA - Hyperelasticity - Uniaxial and biaxial tensile tests $\cdot$ Material parameter identification

\section{Introduction}

Laminated safety glass has become an indispensable component in building construction, automotive and solar industry. It consists of at least two glass panes or glazing materials made of plastic, that are laminated together with a polymeric interlayer. The most known advantages of such compounds are the restraint of glass 
fragments by the polymer film and the ensuring of a certain residual load-bearing after glass breakage, which both reduce the risk of injury. Mechanically speaking, the polymeric interlayer enables a shear transfer between the two glass panes in the intact state. However, here, the difficulty lies in the understanding of the real shear transmission, since the shear modulus shows a polymer-typical strong dependency on temperature and loading time.

Recent research with a focus on the time- and temperature-dependent bearing behaviour of laminated glass undergoing small deformations was conducted by Schuler (2003), Ensslen (2005), Kasper (2005) and Sackmann (2008) using creep and relaxation tests. Further research on the time- and temperature dependent interlayer behaviour using the 'Dynamical-Mechanical-Thermal-Analysis' (DMTA) was done in Barredo et al. (2011), Hooper (2011), Kothe (2013) and Kuntsche (2015). Viscoelastic material models for the interlayer material undergoing small deformations are given in Schuler (2003), Ensslen (2005), Sackmann (2008), Barredo et al. (2011), Hooper (2011) and Kuntsche (2015). A commonly used model is the Generalized Maxwell Model, which is mathematically described by the 'Prony-series' and is implemented in many state-of-the-art FiniteElement-Analysis-Software to incorporate linear viscoelastic material behaviour. The temperature dependency can be taken into account by means of the 'TimeTemperature-Superposition-Principle' (TTSP) in the case of thermorheological simple materials.

Current standards deal very differently with the time and temperature dependency of the interlayer material. While many standards such as the German (DIN 18008 2010) completely neglect the load bearing capacity of the interlayer and solely consider two limit cases (i.e. 'no shear transfer', which considers two separated glass panes and 'full shear transfer', which considers the laminate as a monolithic glass), a few standards such as the NEN 2608 (2014) provide a very general viscoelastic constitutive law in terms of a Prony-series for standard Polyvinylbutyral (PVB) and Sentry Glas ${ }^{\circledR}$.

To overcome the situation in countries with no standardised viscoelastic constitutive law for the time and temperature dependency, some interlayers have general approvals, e.g. Du Pont de Nemours GmbH (2011) and Bridgestone Industrial Limited (2014) for Germany. They propose applicable shear moduli for different load situations in order to approach the real load-bearing capacity of laminated glass, which lies between the two limit cases for the shear transfer and hence enable a much more economic design of glass constructions.

Another aspect in the design of laminated safety glass is the post-breakage behaviour, which leads the interlayer to undergo large deformations. Hence hyperelastic material laws for the constitutive modelling of nonlinear stress-strain phenomena are needed. Recent research in that field was conducted by Bennison et al. (2005) for ionomeric interlayers and by Bennison et al. (2005) and Hooper (2011) for PVB interlayers.

This paper first introduces und summarizes the theoretical background needed for the constitutive modelling of linear viscoelasticity (Sect. 2) and hyperelasticity (Sect. 3). In Sect. 2 recent findings w.r.t. the identification of material parameters of the viscoelastic material model are highlighted. The main part of this paper, Sect. 4, is concerned with the description of the preparation, conduction, documentation and interpretation of various experimental investigations. These investigations were done in order to identify the viscoelastic and hyperelastic constitutive behaviour of a cross-linked ethylene vinyl acetat (EVA) — based interlayer, which is assigned to the groups of elastomers and mostly used in solar industry. In a finalizing section future research activities and first ideas in the field of coupling hyperelastic and viscoelastic constitutive behaviour as well as the incorporation of thermorheological complex material behaviour are highlighted.

\section{Linear viscoelasticity}

\subsection{Generalized Maxwell Model}

\subsubsection{Time domain}

The shear modulus of polymeric interlayers depends on the load duration and load history. The following explanation refers to the simple shear state with the deformation $\gamma$ and the stress state $\tau$ in a strain controlled measurement. When deformed with a constant deformation $\gamma_{0}$, the stress $\tau$ decreases over time and approaches a certain limit value $\tau_{\text {lim }}$ (Fig. 1). This behaviour is called viscoelastic.

In intact laminated safety glass, the large deformation induced material nonlinearity (hyperelasticity, c.f. Sect. 4) is usually not of importance and the theory of linear viscoelasticity can be applied. In literature, the limit for linear viscoelasticity for stiff polymers is given 
Fig. 1 Viscoelastic material behaviour, adapted from Kraus and Niederwald (2016)
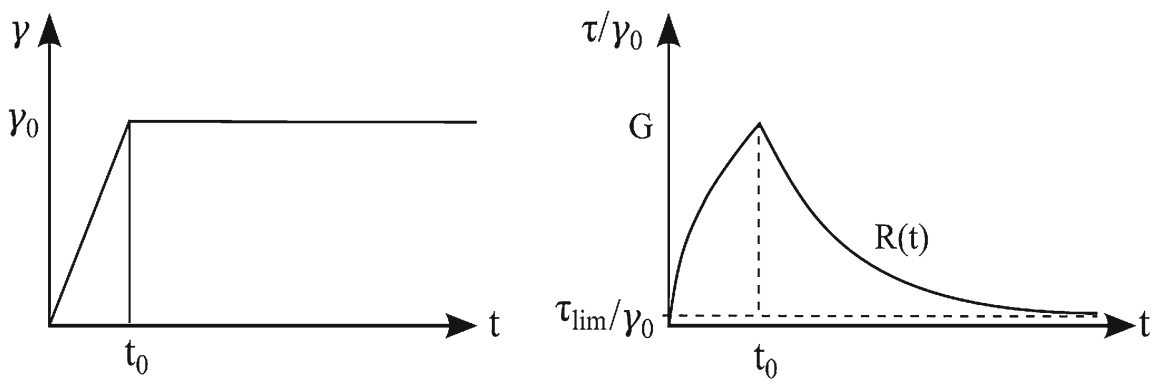

by a strain of $1 \%$ (Grellmann and Seidler 2011), for softer polymers this limit can be much higher (Schwarzl 1990). In the linear viscoelastic region, stress and strain behave linear at a certain time $t$, which can be visualized in isochronous stress-strain diagrams. This means that the stress relaxation function $G(t)=\tau(t) / \gamma_{0}$ is independent of the applied strain amplitude.

After analysing the relaxation modulus as the stress output for a constant strain input, the question arises how to calculate the stress output given an arbitrary strain input. One possibility regarding linear viscoelastic materials is to use the so called 'Boltzmann superposition principle' (Schwarzl 1990), which is sometimes referred to as Duhamel's integral (especially in the dynamics literature).

According to this principle the material response to arbitrary loading processes can be calculated by the sum of material responses obtained under constant loading increments which yields for the limit of infinitesimal increment steps $\Delta s \rightarrow 0$ in Eq. (1).

$\tau(t)=\int_{0}^{t} G(t-s) \frac{\mathrm{d} \gamma}{\mathrm{d} s} d s$

where $G$ : stress relaxation function, $s$ : time with known strain history.

The generalized Maxwell Model in Fig. 2 represents the rheological model of stress relaxation $G(t)$. It consists of $K$ parallel spring-damper elements and a supplementary parallel linear elastic spring. Thus the total stress $\tau$ consists of two parts: the equilibrium stresses, which are described by the equilibrium spring, and the non-equilibrium stresses, which are described by the Maxwell-Elements [Eq. (2)].

$\tau(t)=\int_{0}^{t}\left[G_{\infty}+\sum_{k=1}^{K} G_{k} \exp \left(-\frac{t-s}{\tau_{k}}\right)\right] \dot{\gamma} d s$

wherein $\tau_{k}=\eta_{k} / G_{k}$ is called relaxation time of the $k^{\text {th }}$ element.

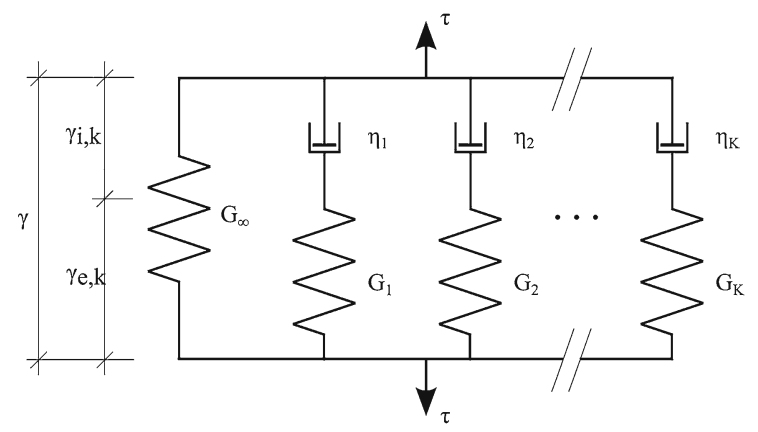

Fig. 2 Generalized Maxwell Model, adapted from Kraus and Niederwald (2016)

The relaxation function given in Eq. (3)

$G(t)=G_{\infty}+\sum_{k=1}^{K} G_{k} \exp \left(-\frac{t}{\tau_{k}}\right)$

is called Prony-series, wherein $G_{k}, \tau_{k}$ and $G_{\infty}$ are called Prony-parameters. They have to be determined through experimental investigation. Every springdamper element has an initial stiffness of $G_{k}$ and relaxes exponentially with a corresponding relaxation time $\tau_{k}$. The additional parallel linear elastic spring characterizes the stiffness at infinite time, respectively the stiffness after relaxation of all spring-damper elements. $G_{\infty}$ equals zero when the considered material shows flowing. Otherwise, it tends to a finite positive limit.

A graphical representation of the combination of four decaying exponentials to a Prony-series is given as an example in Fig. 3. By inspection of Fig. 3 it is remarkable to note, that each Maxwell Element (in terms of the representation by the decaying exponential) mainly decays within one time decade (measured in a logarithmic scale). Thus the total number of Maxwell Elements $K$ has to be chosen such, that the Prony-series represents the experimentally determined time-dependent shear modulus accurately. So far, it has 


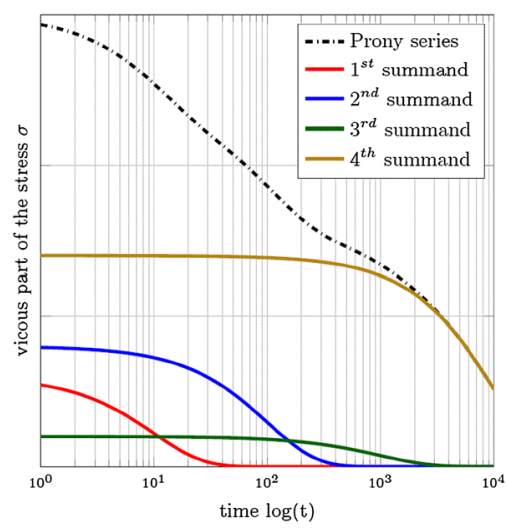

Fig. 3 Principal sketch of a Prony-series and its individual summands, from Kraus and Niederwald (2016)

proven successful to adapt $K$ to the number of investigated time decades.

Other common ways of presenting a Prony-series are:

- Using normalized parameters $g_{k}=G_{k} / G_{0}$, where $G_{0}$ represents the shear modulus of the Generalized Maxwell Model at the time $t=0$ as shown in Eq. (4),

$$
G(t)=G_{0}\left(1-\sum_{k=1}^{K} g_{k}\left(1-\exp \left(-\frac{t}{\tau_{k}}\right)\right)\right)
$$

- In terms of an inner product as presented in Eq. (5).

$$
\begin{aligned}
G(t) & =G_{\infty}+\sum_{k=1}^{K} G_{k} e^{-\frac{t}{\tau_{k}}} \\
& =\left(\begin{array}{c}
G_{\infty} \\
G_{1} \\
\vdots \\
G_{K}
\end{array}\right)^{T} \cdot\left(\begin{array}{c}
1 \\
\exp \left(-t / \tau_{1}\right) \\
\vdots \\
\exp \left(-t / \tau_{K}\right)
\end{array}\right) \\
& =\langle\underline{\boldsymbol{G}}, \underline{\boldsymbol{B}}\rangle
\end{aligned}
$$

where the $G_{\mathrm{k}}$ are the coordinates (summarized in the coordinate vector $\underline{\boldsymbol{G}})$ and the $\underline{\boldsymbol{b}}_{\boldsymbol{k}}=\exp \left(-t / \tau_{k}\right)$ are the basis vectors (summarized in the common basis vector $\underline{\boldsymbol{B}}$.

\subsubsection{Frequency domain}

The time dependent relaxation properties of a material, c.f. Eq. (3), are linked to the complex moduli via the Fourier transform and vice versa (Fig. 4, wherein $\omega$ : angular frequency)

The Fourier transform of Eq. (11) leads to the complex modulus $\boldsymbol{G}^{*}(\omega)$ given in Eq. (6).

$$
\begin{aligned}
\boldsymbol{G}^{*}(\omega)= & G_{\infty}+\sum_{k=1}^{K} G_{k} \frac{\omega^{2} \tau_{k}^{2}}{1+\omega^{2} \tau_{k}^{2}} \\
& +i \sum_{k=1}^{K} G_{k} \frac{\omega \tau_{k}}{1+\omega^{2} \tau_{k}^{2}} \\
= & G_{0}\left(1-\sum_{k=1}^{K} g_{k}+\sum_{k=1}^{K} g_{k} \frac{\omega^{2} \tau_{k}^{2}}{1+\omega^{2} \tau_{k}^{2}}\right. \\
& \left.+i \sum_{k=1}^{K} g_{k} \frac{\omega \tau_{k}}{1+\omega^{2} \tau_{k}^{2}}\right)
\end{aligned}
$$

The real part $\left(\Re\left(\boldsymbol{G}^{*}\right)=G^{\prime}(\omega)\right)$ of Eq. (6) is called the 'Storage modulus' and the imaginary part $\left(\Im\left(G^{*}\right)=\right.$ $G^{\prime \prime}(\omega)$ ) is called 'Loss modulus'. The form with the normalized parameters, c.f. Eq. (4) respectively Eq. (6) is used in the German general technical approvals of some interlayer materials as well as in Sect. 2.3.1 for the two step parameter identification procedure.

Analogously to Eq. (5) the complex modulus representation allows a formulation as an inner product. For the Storage and Loss modulus this is shown in Eqs. (7) and (8).

$$
\begin{gathered}
G^{\prime}(\omega)=\left(\begin{array}{c}
G_{\infty} \\
G_{1} \\
\vdots \\
G_{K}
\end{array}\right)^{T} \cdot\left(\begin{array}{c}
1 \\
\frac{\omega^{2} \tau_{1}^{2}}{1+\omega^{2} \tau_{1}^{2}} \\
\vdots \\
\frac{\omega^{2} \tau_{K}^{2}}{1+\omega^{2} \tau_{K}^{2}}
\end{array}\right)=\left\langle\boldsymbol{G}, \boldsymbol{B}^{\prime}\right\rangle \\
G^{\prime \prime}(\omega)=\left(\begin{array}{c}
G_{\infty} \\
G_{1} \\
\vdots \\
G_{K}
\end{array}\right)^{T} \cdot\left(\begin{array}{c}
0 \\
\frac{\omega \tau_{1}}{1+\omega^{2} \tau_{1}^{2}} \\
\vdots \\
\frac{\omega \tau_{K}}{1+\omega^{2} \tau_{K}^{2}}
\end{array}\right)=\left\langle\boldsymbol{G}, \boldsymbol{B}^{\prime \prime}\right\rangle
\end{gathered}
$$

$G_{\mathrm{k}}$ are the coordinates (summarized in the coordinate vector $\underline{\boldsymbol{G}}$ ) and $\underline{\boldsymbol{b}}^{\prime}{ }_{k}=\frac{\omega^{2} \tau_{k}^{2}}{1+\omega^{2} \tau_{k}^{2}}$ respectively $\underline{\boldsymbol{b}}^{\prime \prime}{ }_{k}=\frac{\omega \tau_{k}}{1+\omega^{2} \tau_{k}^{2}}$ are the basis vectors (summarized in the common basis vector $\underline{\boldsymbol{B}}^{\prime}$ and $\underline{\boldsymbol{B}}^{\prime \prime}$ ). This inner product representation will be used in Sect. 2.3.2 to define the basic regression problem, which is at the heart of 'GUSTL'.

In the context of physics and engineering causal functions are of greatest interest, as these functions respect the basic physical principle, that the effect cannot appear before the cause. The real and imaginary part 
Fig. 4 Structure of the theory of linear viscoelasticity in the time and frequency domain from Kraus and Niederwald (2016)

of its Fourier-Transform $\mathcal{F}(f(t))$ are interrelated, thus they can be calculated from each other.

$$
\begin{aligned}
& \Re(\omega)=\frac{1}{\pi} \int_{-\infty}^{\infty} \frac{\Im(v)}{\omega-v} d v \\
& \Im(\omega)=-\frac{1}{\pi} \int_{-\infty}^{\infty} \frac{\Re(v)}{\omega-v} d v
\end{aligned}
$$

Equations (9) and (10) are called the 'Kramers-Kronig' relations and are valid if the system behaves linear. For practical considerations, especially in terms of validating the causality of the measured data and for doublechecking the Loss modulus $G^{\prime \prime}(\omega)$ (which is usually measured with more noise than the Storage modulus $\left.G^{\prime}(\omega)\right)$ the approximation of Eq. (10) according to (Kraus et al.) is used [Eq. (11)].

$$
\Im(\omega)=G^{\prime \prime}(\omega)=\frac{2}{\pi}\left(\frac{\partial G^{\prime}(u)}{\partial \ln u}\right)_{u=\omega}
$$

\subsection{Time-Temperature-Superposition-Principle (TTSP)}

Typically viscoelastic material properties of polymers strongly depend on temperature. In the energy elastic area, which occurs at temperatures below the glass transition temperature $\left(\mathrm{T} \ll \mathrm{T}_{\mathrm{g}}\right)$, the glassy, brittle polymers reveal a relatively high stiffness, while at temperatures above the glass transition temperature $\left(T \gg T_{g}\right)$ polymers show a rubber-like behaviour which is characterized by much lower stiffness compared to those in the energy elastic area. This area is referred to as entropy elastic area (Schwarzl 1990; Domininghaus et al. 2008).

Depending on the behaviour in the afore-mentioned areas, polymers can be classified into three main groups. The stiffness of thermoplastics, which have no chemical molecular crosslinks, continuously decreases with increasing temperature. In duroplastics, the individual molecular chains are closely interlinked with each other, which results in a low stiffness drop at high temperatures. Elastomers are widely meshed and show a stiffness increment in the entropy elastic area.
Fourier

Transform

complex modulus $\mathrm{G}^{*}(\omega)$

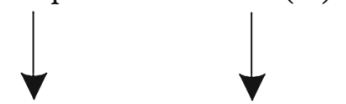

storage modulus $G^{\prime}(\omega)$ loss modulus $G^{\prime \prime}(\omega)$

Since relaxation is based on molecular movements and rearrangement processes, which can be thermally activated, an increase in temperature leads in most cases to an acceleration of the relaxation process. Especially for thermoplastics, this correlation between temperature and load duration is obvious. When all relaxation times have the same dependency on time, which means that the shape of the relaxation curves is identical for all temperatures, the considered material shows a thermorheological simple behaviour. Here, when knowing the relaxation function at a reference temperature $T_{r e f}$, it is sufficient to horizontally shift the curve by the single factor ' $a_{T}$ ' among the time-axis in order to represent the relaxation function for another temperature $T$ [Fig. 5, Eqs. (12) and (13)] (Schwarzl 1990).

$$
\begin{aligned}
\log _{10} a_{T}\left(T, T_{\text {ref }}\right) & =\log _{10} t-\log _{10} t_{\text {ref }} \\
& =\log _{10} \frac{t}{t_{\text {ref }}} \\
G(t, T) & =G\left(a_{T} \cdot t, T_{\text {ref }}\right)
\end{aligned}
$$

The functional dependency of the time dependent shift factors $a_{T}$ can be approximated by commonly used displacement laws. 'Arrhenius' equation [Eq. (14)] is a physical motivated displacement law, which is based on the activation energy that is necessary to activate side chain movements with a certain velocity. Hence, the area of application is limited to the energy elastic region (Brinson and Brinson 2015).

$\log _{10} a_{T}\left(T, T_{r e f}\right)=-0,434 \frac{E_{A}}{R}\left(\frac{1}{T}-\frac{1}{T_{r e f}}\right)$

$E_{A}$ is the activation energy which has to be determined experimentally, $R$ the universal gas constant, $T$ the considered temperature and $T_{\text {ref }}$ the reference temperature in $[K]$.

In the glass transition and entropy elastic area the molecular movement is extended from small side chain movements to full rotations and position changes. Here, the empirical 'William-Landel-Ferry' (WLF) displacement function shown in Eq. (15) delivers more suitable shift factors (Stommel et al. 2011). 
$\log _{10} a_{T}\left(T, T_{r e f}\right)=-\frac{C_{1}\left(T-T_{r e f}\right)}{C_{2}+T-T_{r e f}}$

The two material parameters $C_{1}$ and $C_{2}$ have to be determined experimentally. $T$ is the considered temperature and $T_{\text {ref }}$ is the reference temperature in $\left[{ }^{\circ} \mathrm{C}\right]$. In this context it is worth noticing, that the two material parameters $C_{1}$ and $C_{2}$ are in value strongly dependent on the choice of $T_{\text {ref }}$.

The Arrhenius and WLF functions are only two example functions that represent the temperaturedependency of the shift factor $a_{T}\left(T, T_{\text {ref }}\right)$. Other approaches such as the Arc-tangent (Keuerleber 2006), exponential (Göhler 2010), polynomial (Kuraray Europe GmbH Division TROSIFOL 2014) or the Narayanaswamy-Tool function (available in ANSYS) exist as well in literature.

This TTSP is especially handy for shortening experimental efforts. As time and temperature are correlated by a TTSP law, e.g. WLF, tests with different temperatures can be conducted in a much shorter period of time compared to isothermal relaxation or creep tests. By shifting the single measured isothermal curve sections on the time scale, as illustrated in Fig. 5, a so called 'Master Curve' is obtained, which maps the long-term behaviour (Schwarzl 1990).

\subsection{Prony-parameter-identification}

\subsubsection{Two step data fit}

This paper only gives a brief summary of the in Kuntsche (2015) and Kuntsche et al. (2015) developed two step data fit.

The so-called Prony-parameters $\left(g_{k}, G_{\infty}, \tau_{k}\right)$ of the Generalized Maxwell Model have to be identified in a way that they fit simultaneously the experimentally generated Master Curves of the Storage and Loss modulus from DMTA and TTSP.

This represents a non-linear optimization problem with $2 K+1$ unknowns, where $K$ is the number of spring-damper elements in the Generalized Maxwell Model. With the knowledge that one Maxwell Element per measured frequency decade is sufficient to map the relaxation behaviour, the number of spring-damper elements $K$ equals the number of examined frequency decays. Additionally, this allows to set a relaxation time $\tau_{i}$ to a certain positive value per decade, and hence to reduce the number of unknown parameters to $K+1$.

These can be determined by a least square optimization procedure, wherein the target function is given in Eq. (16). The following basic thermodynamic principles are taken into account during parameter identification:

$$
\begin{aligned}
& -0 \leq g_{k}<1 \\
& -\sum_{k=1}^{K} g_{k} \leq 1 \\
& -G_{0}>0 \text { and } G_{\infty}=G_{0}\left(1-\sum g_{k}\right)
\end{aligned}
$$

Since the values of the moduli spread over a wide range, the target function is formed in the logarithmic region. This has the advantage that the high and low values have a similar influence on the error square. In addition, the Loss modulus is weighted 10 times higher than the Storage modulus in order to take the oscillation of the Loss modulus into account (Göhler 2010).

$$
\begin{aligned}
& f\left(g_{k}, \ldots, g_{K}, G_{\infty}\right) \\
& =\sum_{j=1}^{m}\left[\left(\log G^{\prime}\left(\omega_{j}\right)-\log \bar{G}^{\prime}\left(\omega_{j}\right)\right)^{2}\right. \\
& \left.\quad+10\left(\log G^{\prime \prime}\left(\omega_{j}\right)-\log \bar{G}^{\prime \prime}\left(\omega_{j}\right)\right)^{2}\right]
\end{aligned}
$$

where:

$G^{\prime}\left(\omega_{j}\right)$ : Storage modulus calculated with real part of Eq. (6)

$\bar{G}^{\prime}\left(\omega_{j}\right)$ : Storage modulus out of experiment

$G^{\prime \prime}\left(\omega_{j}\right)$ : Loss modulus calculated with imaginary part of Eq. (6)

$\bar{G}^{\prime \prime}\left(\omega_{j}\right)$ : Loss modulus out of experiment

The optimization problem was implemented in MATLAB as a two-step procedure. In a first step, a genetic algorithm is used to generate suitable start parameters, which are then transmitted in a global gradient based optimization tool (Fig. 6).

Figure 7 presents exemplarily the behaviour of the two-step algorithm for the fit of the Storage and Loss moduli for a given data set (in this case PVB-based interlayer).

\subsubsection{GUSTL data fit}

In the context of this paper only a short summary of the assumptions and the whole deduction of GUSTL (Kraus et al.) can be given.

The elaboration of GUTSL was mainly motivated by the circumstance, that there hardly exists a method of 
Fig. 5 Time-TemperatureSuperposition-Principle, from Kuntsche (2015)
Fig. 6 Two-step optimization algorithm

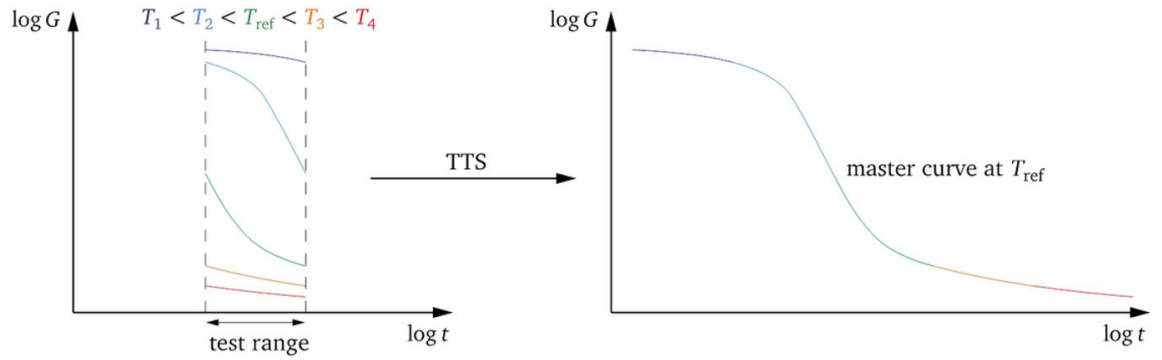

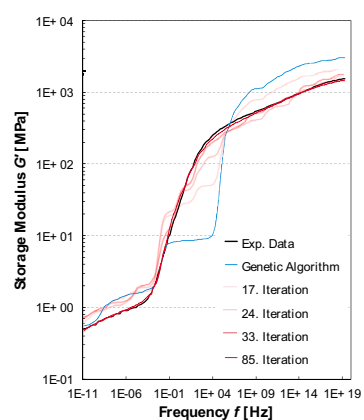

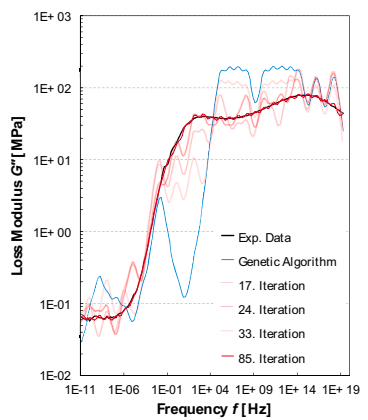

Fig. 7 Principle of two-step optimization algorithm (Kuntsche 2015)

numerically determining the Prony-series from DMTA data, which takes into account the physical relations of and between the Storage and Loss modulus and is not a pure (and / or randomized) data fitting procedure. Indeed, GUSTL is based on picking up the considerations made in Sect. 2.1.2 to set up the determination of the Prony-series as an inverse problem in terms of a matrix-vector product [Eq. (17)]. possessing a righthand side which are the data (Fig. 8).

$\underline{\underline{K}}_{\text {global }} \cdot \underline{\boldsymbol{G}}=\underline{\tilde{\boldsymbol{G}}}$

where

- $\underline{\underline{K}}_{\text {global }}$ is the $N \times K$ dimensional 'global stiffness matrix' containing basis vectors $\underline{\boldsymbol{b}}_{k}^{\prime}$ and $\underline{\boldsymbol{b}}^{\prime \prime}{ }_{k}$
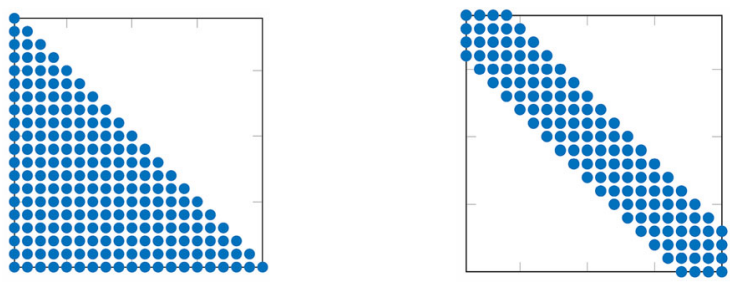

Fig. 8 Principal matrix structures of $\underline{\underline{K}}_{\text {Storage }}$ (left) and $\underline{\underline{K}}_{\text {Loss }}$ (right) from Kraus and Niederwald (2016)

- $\underline{G}$ is the the $K$-dimensional coordinate vector (i.e. the Prony-parameters)

- $\underline{\tilde{G}}$ contains the measured data (for Storage and Loss moduli $G^{\prime}$ and $G^{\prime \prime}$ )

$\tau_{k}=10^{m+k}$ is preset, when the data frequency axis starts at decade $m$.

The global stiffness matrix is constructed out of a part resulting from the Storage modulus and another part coming from the Loss modulus as shown in Eq. (18).

$\underline{\underline{\boldsymbol{K}}}_{\text {global }}=\left[\underline{\underline{\underline{K}}}_{\text {Storage }}, \underline{\underline{K}}_{\text {Loss }}, \mathbf{1}\right]^{T}$

The last line in $\underline{\underline{K}}_{\text {global }}$ ensures the scaling constraint $\sum G_{k}=G_{0}-G_{\infty}$ 
Fig. 9 GUSTL Algorithm

\begin{tabular}{|rl|}
\hline Algorithm 3: GUSTL \\
\hline 1. & Fix the number $K$ of spring-damper elements \\
\hline 2. & Set upper and lower bound for frequency window of investigation $\left[f_{l b}, f_{u b}\right]$ \\
\hline 3. & Set $G_{\infty}$ and $G_{0}$ based on experimental data \\
\hline 4. & Assemble stiffness matrices for the Storage modulus $\underline{\underline{\boldsymbol{K}}}_{\text {Storage }}$ and the Loss modulus $\underline{\underline{\boldsymbol{K}}}_{\text {Loss }}$ \\
\hline 5. & Assemble global stiffness matrix out of $\underline{\underline{\boldsymbol{K}}}_{\text {Storage }}$ and $\underline{\underline{\boldsymbol{K}}}_{\text {Loss }}$ and the scaling constraint \\
\hline 6. & Assemble the right hand side (i.e. the data vector) \\
\hline 7. & Solve the matrix-vector-problem via a non-negative least-squares routine \\
\hline 8. & Result: Final Prony-parameters \\
\hline
\end{tabular}

The stiffness matrix for the Storage modulus $\underline{\underline{K}}_{\text {Storage }}$ possesses the structure of a lower-triangular matrix in case of one Maxwell-Element per decade and is constant one decade after its characteristic relaxation frequency. On the diagonal the contribution of each $\boldsymbol{b}_{\mathrm{k}}^{\prime}$ is equal to $\boldsymbol{b}_{k}^{\prime}\left(1 / \tau_{k}\right)=1 / 2$. The stiffness matrix for Loss modulus $\underline{\underline{K}}_{\text {Loss }}$ is a heptdiagonal matrix in case of one Maxwell-Element per decade.

The principal structure of $\underline{\underline{K}}_{\text {Storage }}$ and $\underline{\underline{K}}_{\text {Loss }}$ is depicted in Fig. 8.

The 'right hand side' vector $\underline{\tilde{G}}$ contains the measured data in the Storage and Loss modulus at the frequencies $\omega_{j}$. Only the last line is not a measurement datum but incorporates the constraint $\sum G_{k}=$ $G_{0}-G_{\infty}$ as shown in Eq. (19).

$\underline{\tilde{\boldsymbol{G}}}=\left[\underline{\tilde{\boldsymbol{G}}}_{\text {Storage }}, \underline{\tilde{\boldsymbol{G}}}_{\text {Loss }} ; \tilde{G}_{0}-\tilde{\boldsymbol{G}}_{\infty}\right]^{T}$

As there is a physical meaning assigned to the entries of the coefficient vector $\underline{G}$ a non-negativity constraint has to be introduced in the solution process of Eq. (19). This is why a 'non-negative least squares' (NNLS) optimization procedure is used to solve Eq. (19) in a physically correct manner (Fig. 9).

\section{Hyperelasticity}

As explained before, the time and temperature dependent shear transmission behaviour of polymers undergoing small deformations and hence showing a linear viscoelastic behaviour can be described by the rheological Generalized Maxwell Model, which concludes to the so called Prony-series and one or more corresponding TTSP to shift the time dependent shear modulus to other temperatures. However, undergoing large deformations, as they occur for example in the fractured state of laminated safety glass, polymeric materials show in addition to the time- and temperature dependent behaviour a non-linear relation between stress and strain expressed in this paper by a hyperelastic material model.

\subsection{Basic continuum mechanics}

Continuum mechanics is based typically on the triplet of

- kinematics,

- balance laws,

- constitutive equations.

In the following, a further elaboration of the kinematics and a discussion of constitutive equations is given, a closer look on the balance laws is omitted, as details on that can be found in e.g. Brinson and Brinson (2015), Marques and Creus (2012) and Tschoegl (1989).

\subsection{Kinematics}

Kinematics is the description of motion and deformation of a material body regardless of the forces applied for that motion, c.f. Tschoegl (1989). In the context of kinematics, the material body consists of an infinite number of material points or particles. In order to find a mathematical description of the kinematics, configurations are introduced. A configuration refers to the simultaneous position of all material points of the body $\mathfrak{B}$. Typically capital and lower case letters indicate, whether quantities belong to the initial (upper case letters, e.g. $X=x_{0} ; t=t_{0} ; \mathfrak{B}_{0}$ ) or the current / deformed configuration (lower case letters, e.g. $x_{t} ; t>t_{0}, \mathfrak{B}$ ) respectively. The bijective deformation map $\chi(X, t)$ defines the position within the current configuration of the particle $P$ that originally occupied a given position $P_{0}$ within the reference configuration. The deformation gradient $\boldsymbol{F}$ [Eq. (20)] describes this movement and is thus a second order tensor.

$\boldsymbol{F}=\operatorname{Grad}\left(\boldsymbol{x}_{\boldsymbol{t}}\right)$

For rubber-like materials incompressibility is considered, which results in $\operatorname{det}(\boldsymbol{F})=J=1$. The left and 
right Cauchy-Green stretch tensors can be derived by Eq. (21).

$\boldsymbol{b}=\boldsymbol{F} \boldsymbol{F}^{T}$ and $\boldsymbol{C}=\boldsymbol{F}^{T} \boldsymbol{F}$

The material models of hyperelasticity are usually formulated in the so called 'Helmholz free energy' (per volume) $\Psi(\boldsymbol{b})$ or $\Psi(\boldsymbol{C})$, which is depending on the principal stretches $\lambda_{i}$, with $i=1,2,3$.

\subsection{Constitutive equations for isotropic hyperelasticity}

Generally speaking, hyperelasticity is a generalization of linear isotropic elasticity, which is able to capture non-linear and large-strain responses of materials exposed to stress. The basic assumption of constitutive models in hyperelasticity is, that the stress function of a thermoelastic material only depends on the applied deformation, the temperature $\theta_{0}$ and the gradient of the temperature $\operatorname{Grad}\left(\theta_{0}\right)$, c.f. Tschoegl (1989).

$$
\begin{aligned}
\boldsymbol{\sigma}_{\text {true }} & =\hat{\boldsymbol{\sigma}}\left(\boldsymbol{F}, \theta_{0}, \operatorname{Grad}\left(\theta_{0}\right)\right) \\
& =\frac{1}{J} \frac{\partial \Psi\left(\boldsymbol{F}, \theta_{0}\right)}{\partial \boldsymbol{F}} \boldsymbol{F}^{\boldsymbol{T}} \\
& =\frac{2}{J} \boldsymbol{F} \frac{\partial \Psi\left(\boldsymbol{C}, \theta_{0}\right)}{\partial \boldsymbol{C}} \boldsymbol{F}^{\boldsymbol{T}}
\end{aligned}
$$

It is possible to express the Helmholz free energy in terms of the three invariants of the left and right Cauchy-Green stretch tensors (Eqs. 23-25).

$$
\begin{aligned}
& I_{1}(\boldsymbol{b})=I_{1}(\boldsymbol{C})=\lambda_{1}^{2}+\lambda_{2}^{2}+\lambda_{3}^{2} \\
& I_{2}(\boldsymbol{b})=I_{2}(\boldsymbol{C})=\lambda_{1}^{2} \lambda_{2}^{2}+\lambda_{2}^{2} \lambda_{3}^{2}+\lambda_{3}^{2} \lambda_{1}^{2} \\
& I_{3}(\boldsymbol{b})=I_{3}(\boldsymbol{C})=\lambda_{1}^{2} \lambda_{2}^{2} \lambda_{3}^{2}
\end{aligned}
$$

With these equations the Cauchy stress can be expressed with Eq. (26).

$$
\boldsymbol{\sigma}_{\text {true }}=\frac{2}{J} \boldsymbol{F}\left[\frac{\partial \Psi}{\partial I_{1}} \frac{\partial I_{1}}{\partial \boldsymbol{b}}+\frac{\partial \Psi}{\partial I_{2}} \frac{\partial I_{2}}{\partial \boldsymbol{b}}+\frac{\partial \Psi}{\partial I_{3}} \frac{\partial I_{3}}{\partial \boldsymbol{b}}\right] \boldsymbol{F}^{\boldsymbol{T}}
$$

Further simplifications of the notation of $\sigma_{\text {true }}$ can be obtained using theorems of continuum mechanics, but these deductions are omitted at this point referencing Brinson and Brinson (2015) and Tschoegl (1989) for further considerations.

In the context of this paper, uniaxial tension and biaxial tension tests were conducted. It is possible, to state analytical expressions for the deformation gradient $\boldsymbol{F}$ as well as the left isochoric Cauchy-Green stretch tensor $\boldsymbol{b}$ in terms of the stretch $\lambda$ for both tested deformation modes [Eqs. (27) and (28)].

Uniaxial tension: $\mathbf{F}=\left[\begin{array}{ccc}\lambda & 0 & 0 \\ 0 & \frac{1}{\sqrt{\lambda}} & 0 \\ 0 & 0 & \frac{1}{\sqrt{\lambda}}\end{array}\right]$ and

$$
\mathbf{b}=\left[\begin{array}{ccc}
\lambda^{2} & 0 & 0 \\
0 & \frac{1}{\lambda} & 0 \\
0 & 0 & \frac{1}{\lambda}
\end{array}\right]
$$

$$
\begin{aligned}
\text { Biaxial tension: } & \mathbf{F}=\left[\begin{array}{ccc}
\lambda & 0 & 0 \\
0 & \lambda & 0 \\
0 & 0 & \frac{1}{\lambda^{2}}
\end{array}\right] \text { and } \\
\mathbf{b} & =\left[\begin{array}{ccc}
\lambda^{2} & 0 & 0 \\
0 & \lambda^{2} & 0 \\
0 & 0 & \frac{1}{\lambda^{4}}
\end{array}\right]
\end{aligned}
$$

The deformation gradient $\boldsymbol{F}$ can be decomposed into dilatational and distortional components as shown in Eq. (29).

$\mathbf{F}=J^{1 / 3} \mathbf{F}^{*}$

Thus the invariants can also be decomposed into dilatational and distortional components:

$I_{1}(\boldsymbol{b})=J^{2 / 3} I_{1}^{*}$

$I_{2}(\boldsymbol{b})=J^{4 / 3} I_{2}^{*}$

In the following three subsections, some of the common Helmholz free energy functions are presented, which will be used for the fitting of the material responses obtained in the conducted experiments. It should be noted here, that the three different Helmholz free energy functions in the remainder of this section do include the invariants in different ways and thus are or are not well capable of reproducing the material response due to different testing scenarios from an energetic point of view, as the specific material laws lay different weight to different invariants. The discussion of such considerations is far beyond the scope of this paper but is addressed for example in Drass et al. (2017), Kaliske and Heinrich (1999), Bennison et al. (2005) etc.

\subsubsection{Neo-Hooke}

The Neo-Hookean material model is a simple hyperelastic model based the two material constants shear 
modulus $\mu$ and bulk modulus $\kappa$. The Helmholz free energy function is given in Eq. (32).

$\Psi\left(I_{1}^{*}, J\right)=\frac{\mu}{2}\left(I_{1}^{*}-3\right)+\frac{\kappa}{2}(J-1)^{2}$

The Cauchy stresses for the standard experiments and the assumption of an incompressible material can be calculated via Eqs. (33) and (34).

$$
\begin{aligned}
\sigma_{\text {true, uniaxial }} & =\mu\left(\lambda^{2}-\frac{1}{\lambda}\right) \\
\sigma_{\text {true,biaxial }} & =\mu\left(\lambda^{2}-\frac{1}{\lambda^{4}}\right)
\end{aligned}
$$

\subsubsection{2-Parameter Mooney-Rivlin}

The two parameter Mooney-Rivlin material model is an extension of the Neo-Hooke material model based on three material constants $C_{10}, C_{01}$ and the bulk modulus $\kappa$. The Helmholz free energy function is given in Eq. (35).

$$
\begin{aligned}
\Psi\left(I_{1}^{*}, I_{2}^{*}, J\right)= & C_{10}\left(I_{1}^{*}-3\right)+C_{01}\left(I_{2}^{*}-3\right) \\
& +\frac{\kappa}{2}(J-1)^{2}
\end{aligned}
$$

The Cauchy stresses for the standard experiments and the assumption of an incompressible material can be calculated via Eqs. (36) and (37).

$$
\begin{aligned}
\sigma_{\text {true }, \text { uniaxial }}= & 2\left(\lambda^{2}-\frac{1}{\lambda}\right)\left[C_{10}+\frac{C_{01}}{\lambda}\right] \\
\sigma_{\text {true }, \text { biaxial }}= & 2 C_{10}\left(\lambda^{2}-\frac{1}{\lambda^{4}}\right) \\
& +2 C_{01}\left[\lambda^{4}-\frac{1}{\lambda^{2}}\right]
\end{aligned}
$$

\subsubsection{Extended-tube}

The Extended-Tube (ET) model is a material model, which is inspired by statistical and micro mechanics. It tries to consider the network constraints from surrounding molecular chains and the limited chain extensibility in the derivation of the Helmholz free energy expression, c.f. Kaliske and Heinrich (1999).

The energy function consists of three parts: energy from crosslinking of the network $\Psi_{c}$ [Eq. (38)], energy from confining tube constraints $\Psi_{e}$ [Eqs. (39) and
(41)] and energy from volumetric deformations $\Psi_{v}$ [Eq. (40)]: $\Psi=\Psi_{c}+\Psi_{e}+\Psi_{v}$.

$$
\begin{aligned}
\Psi_{c}= & \frac{G_{c}}{2}\left[\frac{\left(1-\delta^{2}\right) \cdot\left(I_{1}^{*}-3\right)}{1-\left(I_{1}^{*}-3\right) \delta^{2}}\right. \\
& \left.+\ln \left(1-\delta^{2}\left(I_{1}^{*}-3\right)\right)\right] \\
\Psi_{e}= & \frac{2 G_{e}}{\beta^{2}} \sum_{i=1}^{3}\left(\left(\lambda_{i}^{*}\right)^{-\beta}-1\right) \\
\Psi_{v}= & \kappa(J-1)^{2} \\
\lambda_{i}^{*}= & J^{-\frac{1}{3}} \lambda_{i}
\end{aligned}
$$

As we here only consider the incompressible case it follows, that $\Psi_{v}=0$. The material constants are hence $G_{c}, G_{e}, \delta$ and $\beta$. The Cauchy stresses for the standard experiments can be calculated via Eqs. (42) and (43).

$$
\begin{aligned}
& =G_{c}\left\{\frac{1+\left(1+I_{1}^{* 2}-4 I_{1}^{*}\right) \delta^{2}+\left(5 I_{1}^{*}-I_{1}^{* 2}-6\right) \delta^{4}}{\left[1-\left(I_{1}^{*}-3\right) \delta^{2}\right]^{2}}\right\} \\
& \quad\left(\lambda^{2}-\frac{1}{\lambda}\right)-\frac{2 G_{e}}{\beta}\left[\lambda^{-\beta}-\left(\frac{1}{\sqrt{\lambda}}\right)^{-\beta}\right] \\
& \sigma_{\text {true }, B T} \\
& =G_{c}\left\{\frac{1+\left(1+I_{1}^{* 2}-4 I_{1}^{*}\right) \delta^{2}+\left(5 I_{1}^{*}-I_{1}^{* 2}-6\right) \delta^{4}}{\left[1-\left(I_{1}^{*}-3\right) \delta^{2}\right]^{2}}\right\} \\
& \quad\left(\lambda^{2}-\frac{1}{\lambda^{4}}\right)-\frac{2 G_{e}}{\beta}\left[\lambda^{-\beta}-\left(\frac{1}{\lambda^{2}}\right)^{-\beta}\right]
\end{aligned}
$$

\section{Experimental investigations}

The EVA is tested in an already cured state, so that the specimens do not need special conditioning as it would be necessary for e.g. PVB or SentryGlas ${ }^{\circledR}$. Considering the tensile tests in 4.2 and 4.3 , the conditioning and the testing was done at $23^{\circ} \mathrm{C}$ and $50 \%$ relative humidity according to DIN ISO 572-1 (2012). The following results were obtained, assuming incompressible material behaviour.

\subsection{Dynamical-mechanical-thermal-analysis}

\subsubsection{Basic principles of DMTA}

The principle of DMTA is to apply a sinusoidal stress or strain to the specimen and to measure the material 
Fig. 10 Phase lag between strain input and stress response (left time domain, right complex plane) from Kraus and Niederwald (2016)

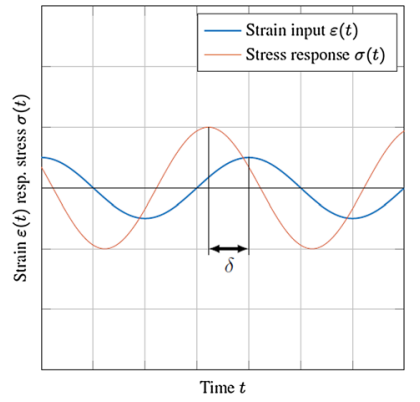

response, c.f. Kraus and Niederwald (2016), Kuntsche (2015). From this, it is possible to determine the stiffness of the material and also its viscosity from the phase-lag $\tan \delta$ between the oscillating stress or strain and the material response (Fig. 10).

In practice, the DMTA testing machine cools or heats to the first testing temperature and then performs a frequency sweep (testing multiple frequencies on the same temperature level) to obtain moduli within a bounded frequency range at a specific temperature, c.f. Kraus and Niederwald (2016), Kuntsche (2015). After the next temperature is tuned the frequency sweep is conducted. This procedure repeats until the whole temperature range of interest is processed. In a next step, the TTSP can be used to extend the bounded frequency range to obtain the complex moduli for several decades and temperatures.

\subsubsection{Description}

The temperature-frequency sweep was performed at TU Darmstadt in a torsion DMTA using a machine 'Anton Paar MCR 702'. One approx. $45 \times 10$ and $0.7 \mathrm{~mm}$ thick EVA sample was extracted and clamped

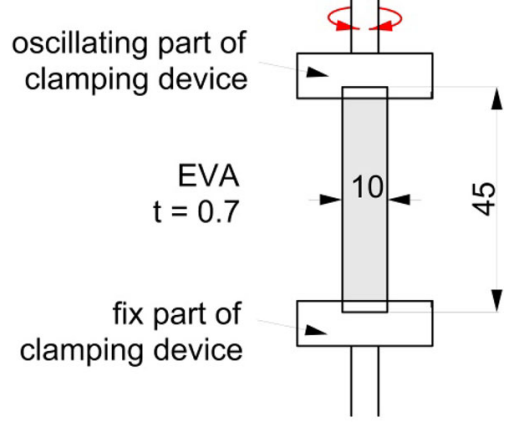

Fig. 11 Geometry of DMTA test specimen; all dimensions in $\mathrm{mm}$ in the measuring system whose lower part is immovable and the upper part oscillates around a resting point, c.f. Figs. 11 and 12. The mechanical vibration is generated by an electronically commutated synchronous motor which simultaneously measures the torque. The angle of rotation and thus indirectly the shear are detected by means of an optical rotary encoder. The measurement was carried out in a displacement controlled way. Accordingly, a shear amplitude of $0.02 \%$ was applied with different frequencies $[f=0.01-50 \mathrm{~Hz}]$ and at different temperatures $\left[T=-80^{\circ} \mathrm{C}\right.$ to $T=+80^{\circ} \mathrm{C}$ in $5^{\circ} \mathrm{C}$ steps]. The corresponding torque or shear stress and the phase lag were measured. From this data, the temperature- and frequency-dependent Storage and Loss moduli are determined and output by the device. The desired temperature is achieved by an annealing system based on convection heating. In addition, liquid nitrogen is evaporated and blown directly into the furnace to reach the very low temperatures.

Prior to the start of the temperature-frequency sweep, amplitude sweeps were performed at two constant temperatures (in the entropy elastic area at $T=$ $-80^{\circ} \mathrm{C}$ and in the energy elastic area at $T=23^{\circ} \mathrm{C}$ )

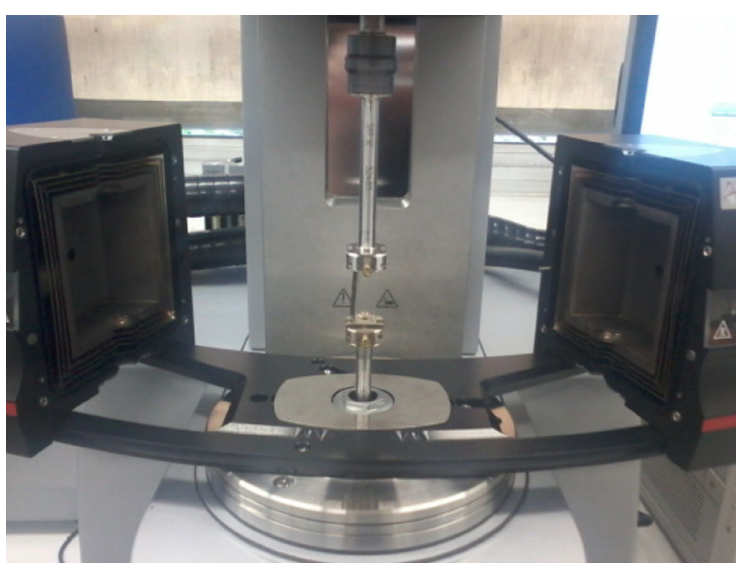

Fig. 12 DMTA test set up 


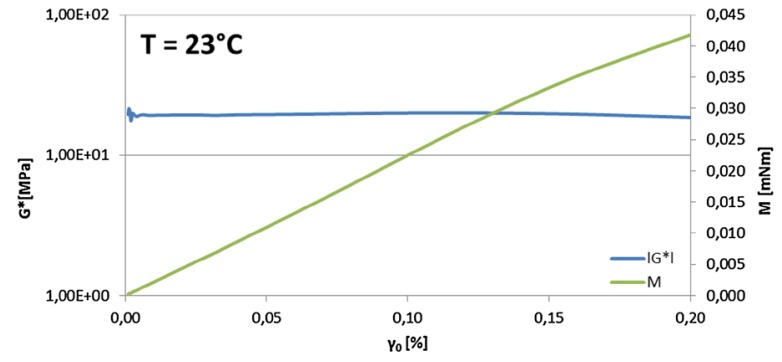

Fig. 13 Linearity check due to amplitude sweep

and a constant frequency of $1 \mathrm{~Hz}$ to ensure that the linear viscoelastic range was not left during the experiments. The applied strain varied between 0.001 and $0.2 \%$. The glass transition temperature has been determined in a temperature sweep $\left[T=-80^{\circ} \mathrm{C}\right.$ to $T=$ $+80^{\circ} \mathrm{C}$, heating rate $1 \mathrm{~K} / \mathrm{min}$ ] with a constant frequency of $1 \mathrm{~Hz}$ and a constant strain amplitude of $0.02 \%$ by means of the maximum value of the Loss factor $\tan \delta=G^{\prime \prime}(\omega) / G^{\prime}(\omega)$ to $T_{g} \sim-20^{\circ} \mathrm{C}$.

\subsubsection{Results}

As stated in the section above, prior to the temperaturefrequency sweeps, amplitude sweeps were performed at two constant temperatures and a constant frequency to ensure linear viscoelastic behaviour of the test samples during the temperature-frequency sweep experiments. The results are depicted in Fig. 13. It can be seen

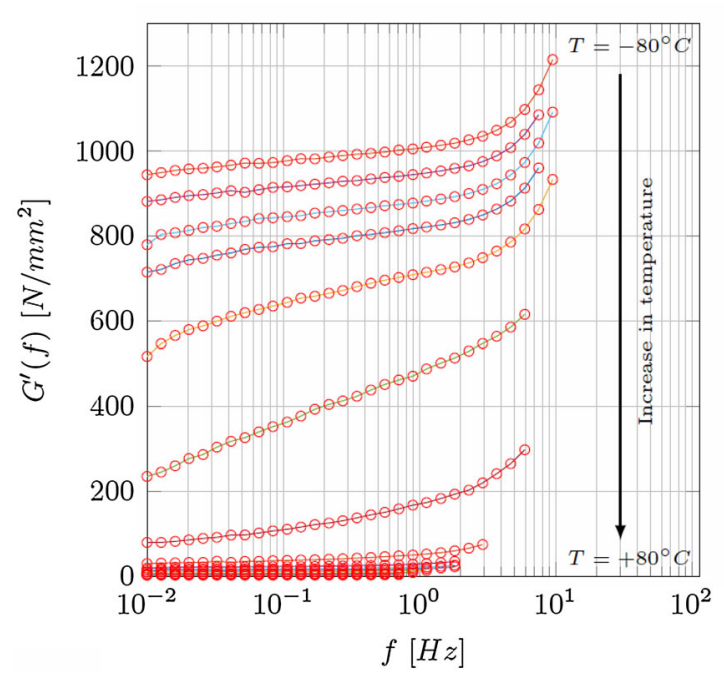

Fig. 14 Storage modulus $\mathbf{G}^{\prime}$ (f) raw data

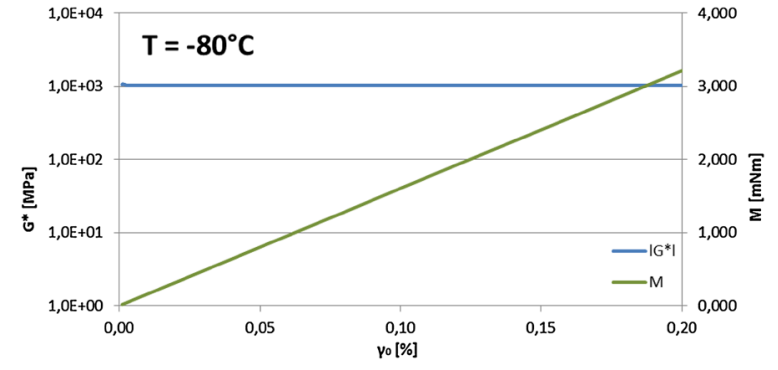

that an approximately constant complex modulus (i.e. magnitude of the complex modulus) occurs for both temperatures above and below the glass transition temperature $\left(\mathrm{T}_{\mathrm{g}} \sim-20^{\circ} \mathrm{C}\right)$ for the applied deformations. This further means that the assumption of a linear viscoelastic material behaviour during the consecutively conducted temperature-frequency sweeps is justified.

The temperature-frequency sweep was conducted on one single specimen in torsional mode with displacement control. A shear amplitude of $0.02 \%$ strain was applied at different frequencies in the range of $f \in$ $[0.01 ; 50] \mathrm{Hz}$ (logarithmically equidistant spaced in 15 intervals) at different temperatures $T \in[-80 ; 80]{ }^{\circ} \mathrm{C}$ in steps of $5^{\circ} \mathrm{C}$.

The raw data are given in Figs. 14 and 15.

Applying a horizontal shifting procedure as described in Sect. 2.2 the Master Curves for the Storage and Loss moduli are obtained. In a next step the two methods as mentioned in Sects. 2.3.1 and 2.3.2 (two-step data fit as

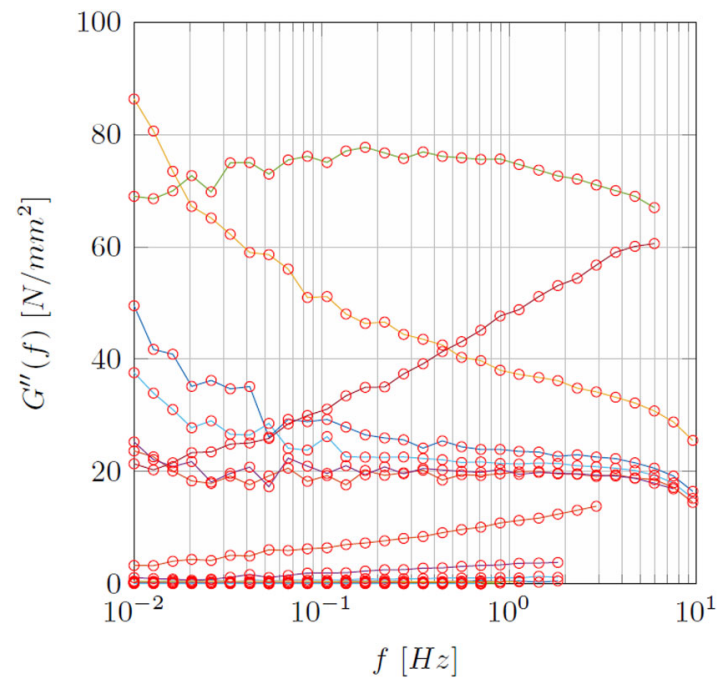

Fig. 15 Loss modulus $\mathbf{G}^{\prime \prime}$ (f) raw data 
Fig. 16 Storage modulus $\mathbf{G}^{\prime}$ (f) calculated by the different methods

Fig. 17 Loss modulus $\mathbf{G}^{\prime \prime}$ (f) calculated by the different methods
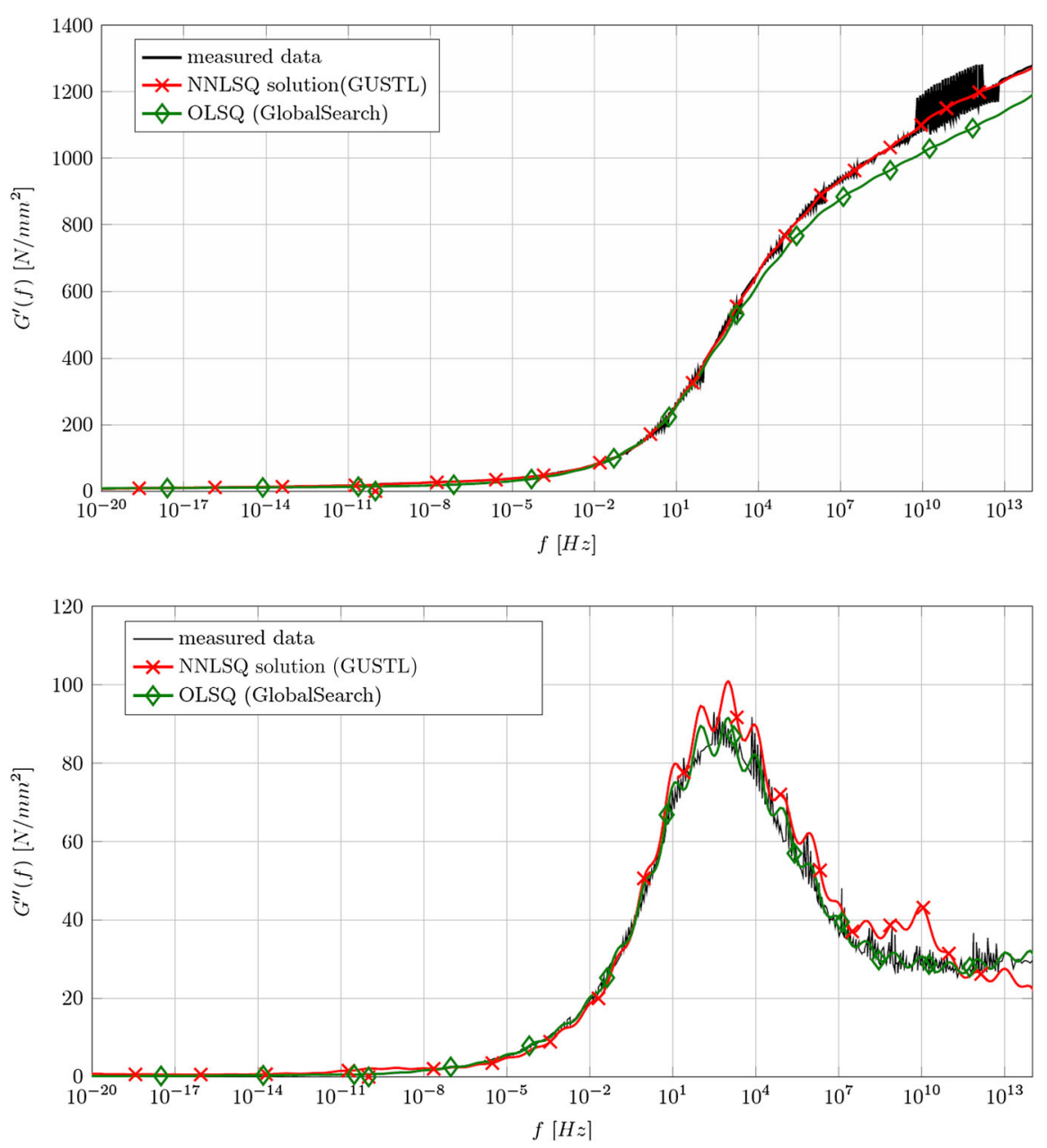

well as GUSTL) are applied to fit a Prony-series to the data from the Master Curves. It was chosen to use 33 relaxation times $\tau_{k}=10^{-20+k}, k=1: 33$ for each fitting method in order to provide one Maxwell-Element per frequency-decade. The results of the fits are given Figs. 16 and 17.

The Master Curves for Storage and Loss moduli are fitted quite well by both methods. In the Storage modulus GUSTL fits the data better whereas in the Loss modulus, the 2-step Global Search method meets the measured data more accurately. This may be due to Eq. (16), which requires a simultaneous fitting of the Shear and Loss Modulus, where the Loss Modulus is weighted 10 times higher in the two-step Global Search fitting procedure.

The distribution of the coefficients $G_{i}$ obtained via the two-step (Global Search) method in comparison to GUSTL is given in Fig. 18.
When comparing the estimated coefficients $G_{i}$ obtained via the two methods, they differ in some regions but deliver in the overall a similar result. It is worth mentioning, that the solution for the coefficients $G_{i}$ when using GUSTL is within milliseconds whereas the solution found by the two-step method takes up to minutes or even hours.

Finally and by referencing the statements made in Sect. 2.1.2, the linearity of the observed data modelled with a Generalized Maxwell-Element by applying Eq. (11) to the Storage modulus Master Curve and approximately validate the obtained Master Curve for the Loss modulus is checked. Here again a theoretical justification for the assumption of having conducted the tests within the bounds of linearity can be given, which is in accordance with the amplitude sweeps presented in Fig. 13. In frequency regions above $10^{7} \mathrm{~Hz}$, the oscillating behaviour of Kramers-Kronig-Approximation for 
Fig. 18 Estimated coefficients $\mathbf{G}_{\mathbf{i}}$ of the Prony-series

Fig. 19 Linearity check via the approximate Kramers-Kronig-Transform of $\mathbf{G}^{\prime}(\mathbf{f})$ against the Loss modulus $\mathbf{G}^{\prime \prime}(\mathbf{f})$
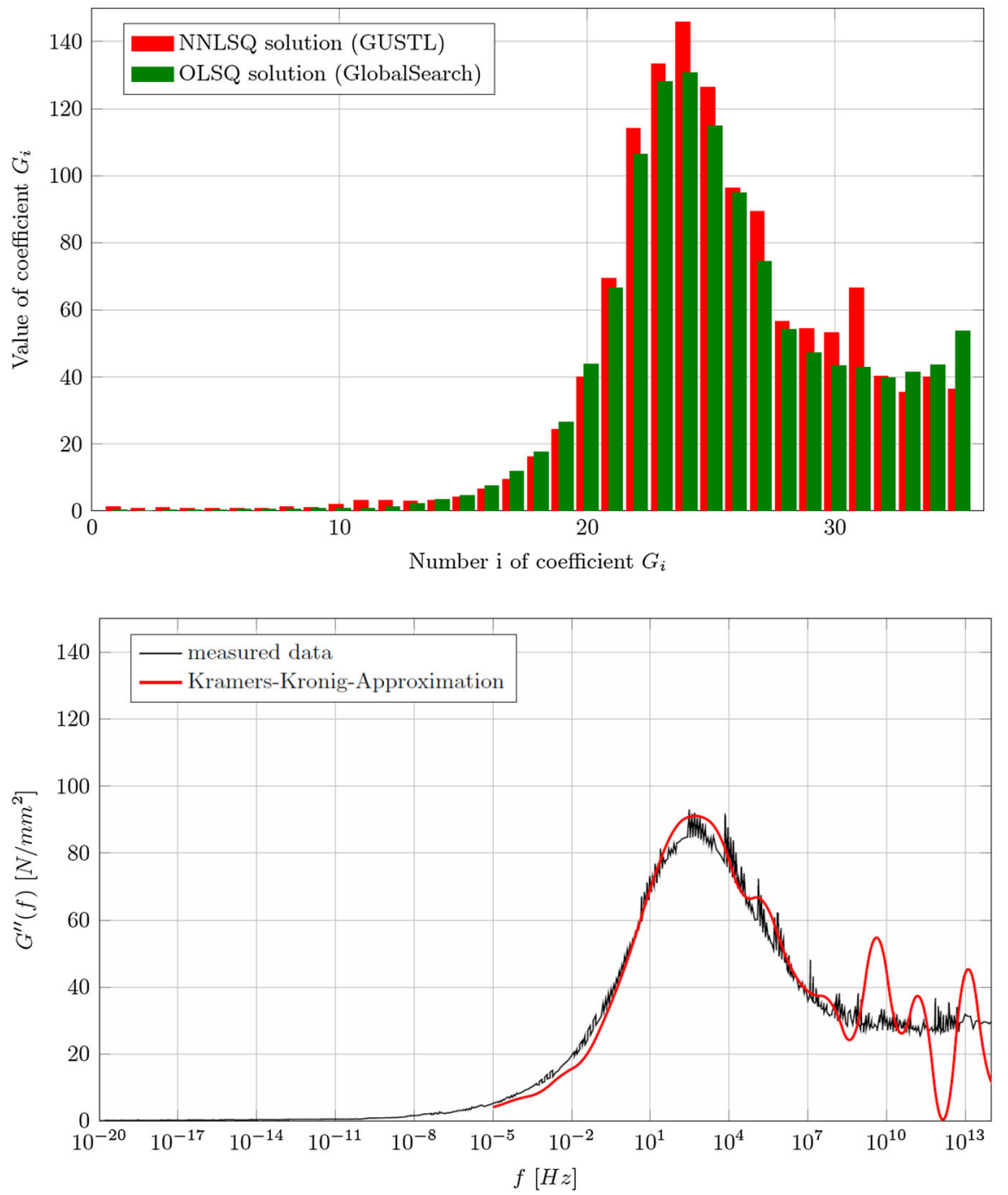

the Loss modulus results from the derivation of the wavy spline approximation of the Storage modulus, c.f. Figs. 16 and 19 and Eq. (11).

\subsection{Uniaxial tensile tests}

\subsubsection{Description UT}

The uniaxial tensile tests (UT) were performed in imitation of ISO 527 at TU Darmstadt with a nonstandardized shape with a relatively short edge-parallel area (Fig. 20) since the goal of the tests was to determine the stress-strain behaviour at different strain rates from quasi-static to dynamic loading (Schneider et al. 2012; Becker 2009).
The presented test procedure and results are restricted in this paper to the investigated EVA at constant quasistatic haul-off velocity $v=50 \mathrm{~mm} / \mathrm{min}$.

Before testing, five $0.7 \mathrm{~mm}$ thick specimens were extracted from the sheet by a CNC drilling machine and red dots were applied for the optical strain evaluation with 'Digital Image Correlation' (DIC). The tests were performed with a universal testing machine (type 'Zwick Z050'). The experiments were driven up to the breaking to detect-in addition to the stress-strain relations - the breaking strain of the EVA. Figure 22 shows a specimen before and after the uniaxial tensile test. It becomes obvious that the EVA shows plastic deformations. Figure 21 shows a specimen in the testing machine. 


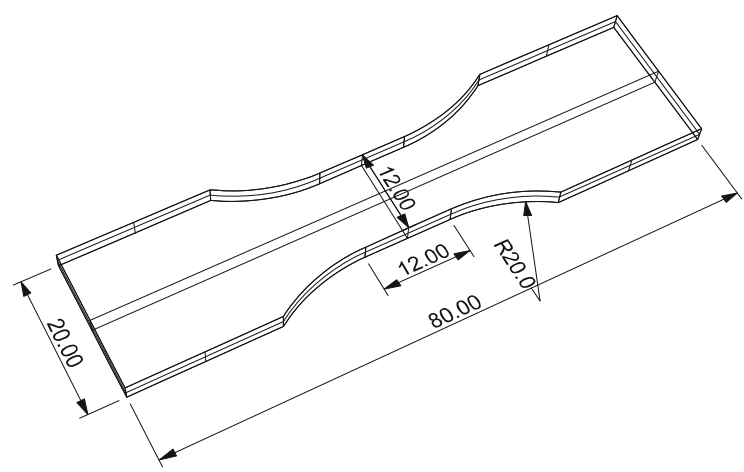

Fig. 20 Geometry of uniaxial tension test specimen; all dimensions in $\mathrm{mm}$

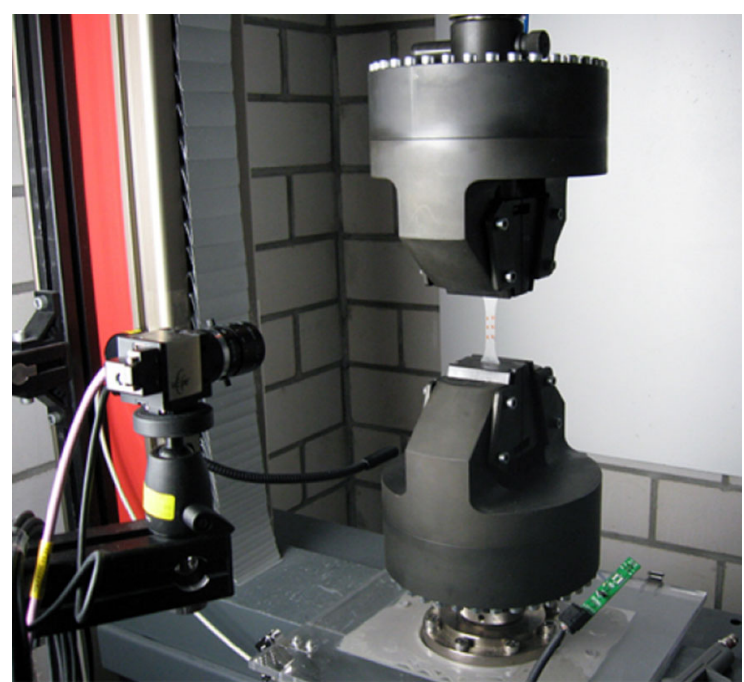

Fig. 21 Uniaxial tensile test set up

The true strain $\varepsilon^{\text {true }}$ [Eq. (44)] was obtained by DIC, the true stress $\sigma^{\text {true }}$ [Eq. (45)] was determined by the force measurement of the universal testing machine and the actual cross section $A_{\text {true }}$, which was backcalculated from the measured strain in transverse direction under assumption of incompressibility.

$$
\begin{aligned}
\varepsilon_{x}^{\text {true }} & =\ln \left(1+\varepsilon_{x}^{\text {eng }}\right)^{2 \cdot v}=\ln \left(1+\frac{\Delta l_{x}}{l_{0, x}}\right) \\
\sigma^{\text {true }} & =\frac{\mathrm{F}}{A_{\text {true }}}
\end{aligned}
$$

\subsubsection{Results $U T$}

The fit of the data out of the UT was conducted via MATLAB in a least-squares setting for the three hyperelastic material models introduced in Sect. 3.3.

For the data fitting of the UT it was necessary to use a suitable initial guess which leads to a good approximation of the material responses for the ExtendedTube (ET) model (which is summarized in Table 1). As indicated by the difference $\Delta \sigma_{\text {true }}$ in Fig. 23 (between the mean true stress value and the fitted true stress) the Neo-Hooke as well as the Mooney-Rivlin material models were not able to approximate the curvature of the measured data in a suitable way whereas the ET material model was.

\subsection{Biaxial tensile test}

\subsubsection{Description BT}

The biaxial tensile tests (BT) were performed at Fraunhaufer EMI with six cross-shaped EVA samples that have been excised from EVA sheets. They provide an area of $A=80 \times 80 \mathrm{~mm}^{2}$, where pure biaxial stress and strain conditions can be guaranteed and $60 \mathrm{~mm}$ long clamping areas (see Figs. 24 and 25). The average thickness of all specimen was measured to $t=0.7 \mathrm{~mm}$.

During BT, the cross-shaped sample (in Fig. 26) was pulled simultaneously in $\mathrm{x}$ - and $\mathrm{y}$-direction. The loadings were applied by four orthogonal oriented actuators with a quasi-static speed of $v=25 \mathrm{~mm} / \mathrm{min}$ per axis, which corresponds to $v_{B T}=50 \mathrm{~mm} / \mathrm{min}$ for both directions $\mathrm{x}$ and $\mathrm{y}$ until failure. The forces required for this were measured with a piezoelectric force sensor
Fig. 22 EVA specimen before and after uniaxial tensile test

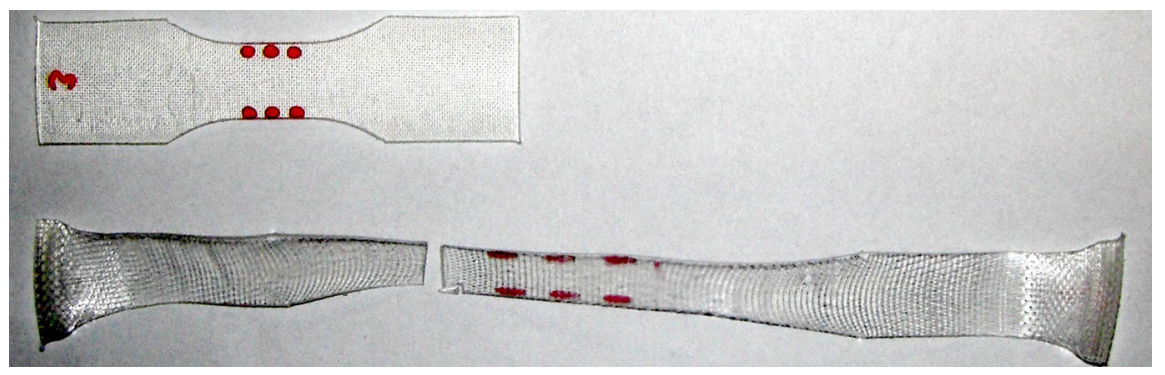



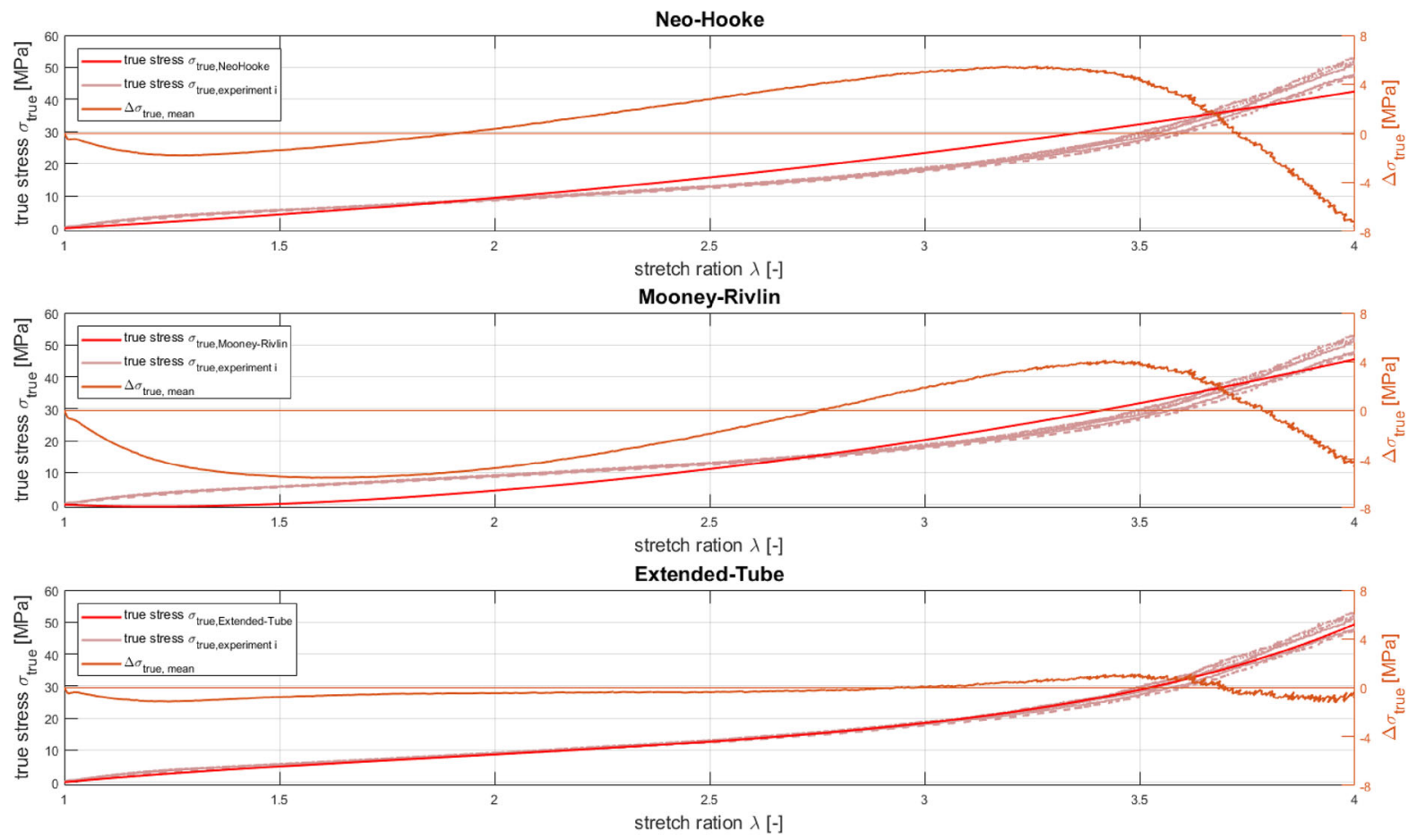

Fig. 23 Fitting different material models to the EVA sample data from the uniaxial tensile test (UT)

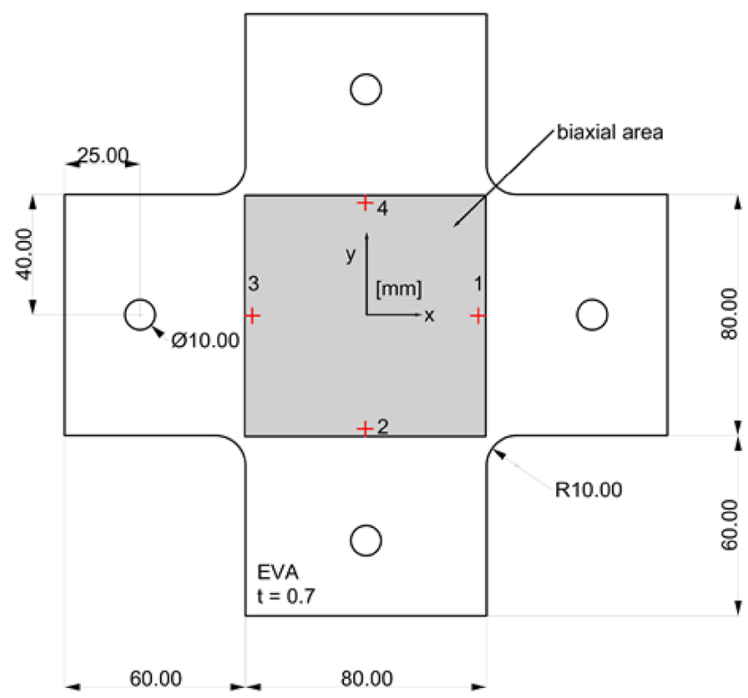

Fig. 24 Geometry of biaxial tensile test specimen; all dimensions in (mm) (Drass et al. 2017)

and the axial movements with magnetic displacement sensors. The elongation per axis during test was measured with a grey-value pattern that has been applied with a water based black-white varnish to the samples and evaluated by DIC.
The evaluation was carried out with the forcedisplacement data, wherein the forces in $\mathrm{x}$-/y-direction were defined as arithmetic mean values of two opposing actuators, which is given by Eq. (46).

$F_{x}=\frac{1}{2} \cdot\left(F_{1}+F_{3}\right)$ and $F_{y}=\frac{1}{2} \cdot\left(F_{2}+F_{4}\right)$

The total local elongations in $\mathrm{x}$ - and $\mathrm{y}$-direction were determined with DIC, wherein the outer points of the grey value pattern of each axis (Fig. 24) were tracked by a video camera during the experiment. The relative elongation can be calculated with Eq. (47).

$\Delta l_{x}=u_{1}+u_{3}$ resp. $\Delta l_{y}=u_{2}+u_{4}$

The obtained data was then used to calculate engineering stresses and strains with Eqs. (48) and (49).

$\varepsilon_{x}^{\text {eng }}=\frac{\Delta l_{x}}{l_{0, x}}$ resp. $\quad \varepsilon_{y}^{\text {eng }}=\frac{\Delta l_{y}}{l_{0, y}}$

$\sigma_{x}^{e n g}=\frac{F_{x}}{b \cdot t} \quad$ resp. $\quad \sigma_{y}^{e n g}=\frac{F_{y}}{b \cdot t}$

From these the true quantities can be determined as shown in Eqs. (50) and (51) for the x-direction.

$\varepsilon_{x}^{\text {true }}=\left[\ln \left(1+\varepsilon_{x}^{\text {eng }}\right)\right]^{2 \cdot v}=\ln \left(1+\varepsilon_{x}^{\text {eng }}\right)$ 


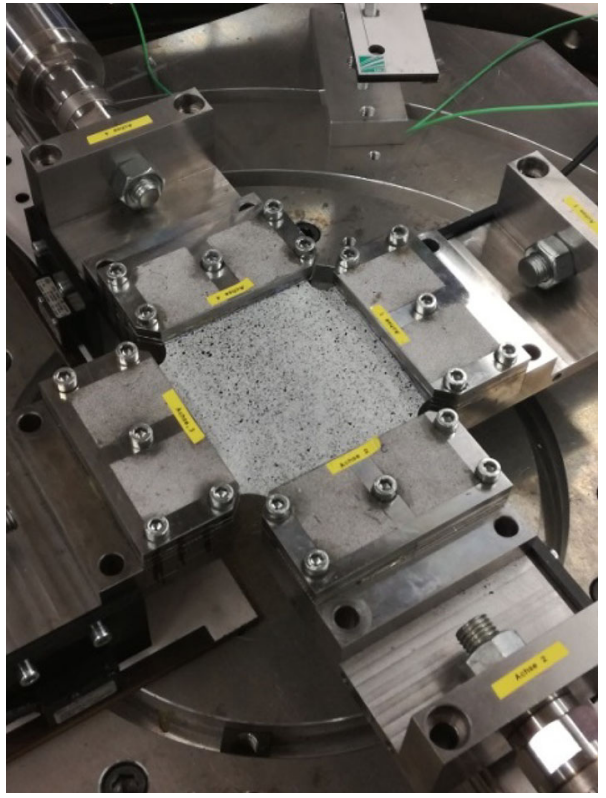

Fig. 25 Biaxial tensile test set up

$\sigma_{x}^{\text {true }}=\left[1+\varepsilon_{x}^{\text {eng }}\right] \cdot \sigma_{x}^{\text {eng }}$

under assumption of incompressibility.

During the evaluation, it could be demonstrated that there is no anisotropy. The stresses and strains are independent of direction, hence $\sigma_{x}=\sigma_{y}$ and $\epsilon_{x}=\epsilon_{y}$.

\subsubsection{Results}

The fit of the data out of the BT was conducted via MATLAB in a least-squares setting for the three hyperelastic material model, introduced in sec. 3.3.

As was obtained in Sect. 4.2.2 for the data fitting of the UT it was necessary to use a suitable initial guess which leads to a good approximation of the material responses for the ET model. Again for the ET model a strong dependency of the fitting on the initial guess of the material parameters was observed (which is summarized in Table 1). In the BT testing mode the NeoHooke model captured the curvature of the data the least. For the Mooney-Rivlin material model as well as for the ET material model a balancing (in terms of over- and underestimating the measured data with the fit) behaviour can be observed, c.f. Fig. 27. Again the least deviation of the fitted model from the measured data can be obtained by the ET material model. Here also the difference $\Delta \sigma_{\text {true }}$ (between the mean true stress value and the fitted true stress) is used as a measure for the goodness of the fit against the measured data.

\subsection{Parameter identification from a combined test data fit}

In the context of this paper a material model parameter identification procedure is presented, which takes into account the data from the two experimental settings (UT and BT). The procedure was also conducted via MATLAB in a least-squares setting for the three hyperelastic material models, introduced in sec. 3.3.

Here it is observed, that the 'simpler' material models Neo-Hooke as well as Mooney-Rivlin were independent of the initial material parameter guess whereas the ET shows a strong dependency of the final estimated material parameters by the least-squares fitting routine. Some results of the fitting are presented in Fig. 28.

In Table 1, the most interesting is, that the combined fit results are very similar (in magnitude) to those obtained by a pure UT test fitting. Also in the combined data fitting process, a suitable initial guess leads to a good approximation of the material responses for both test modes by using the ET model. The Neo-Hooke as well as the Mooney-Rivlin material model were not
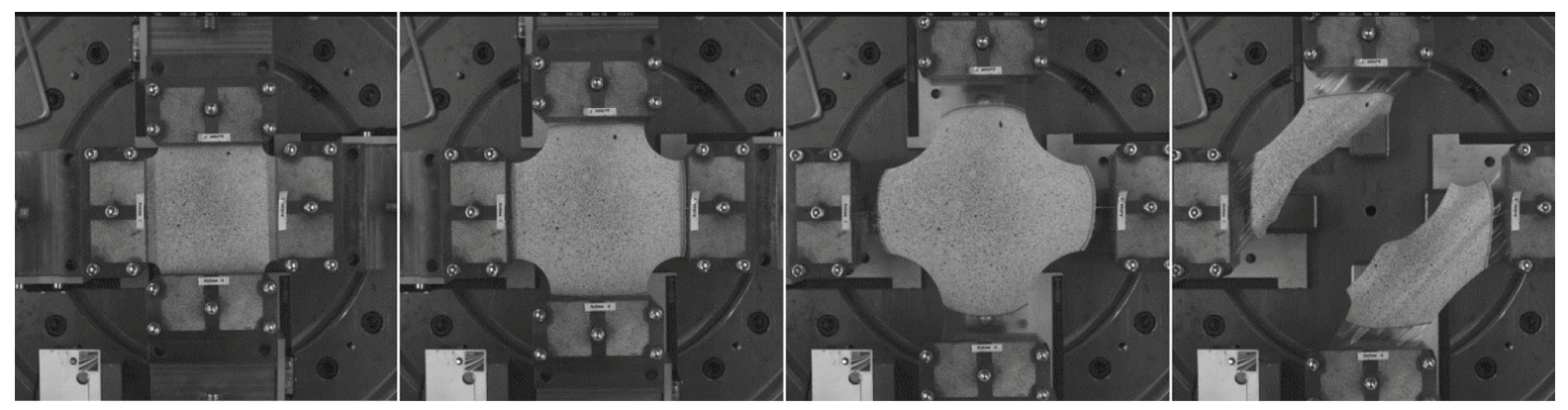

Fig. 26 EVA sample during the biaxial tensile test 
Table 1 Summary table containing the finally fitted material model parameters and the sensitivity of the material model w.r.t. the initial guess of the material parameters for the different test modes UT and BT

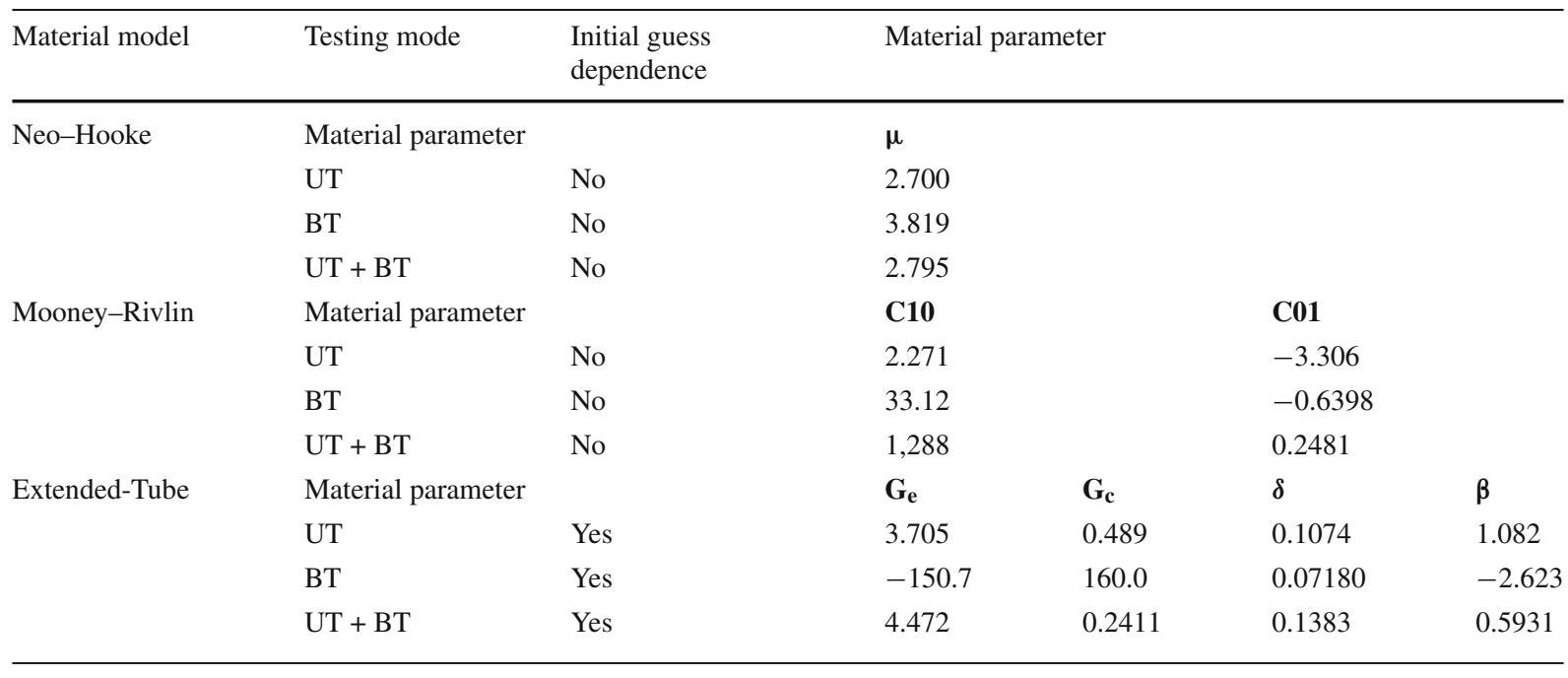
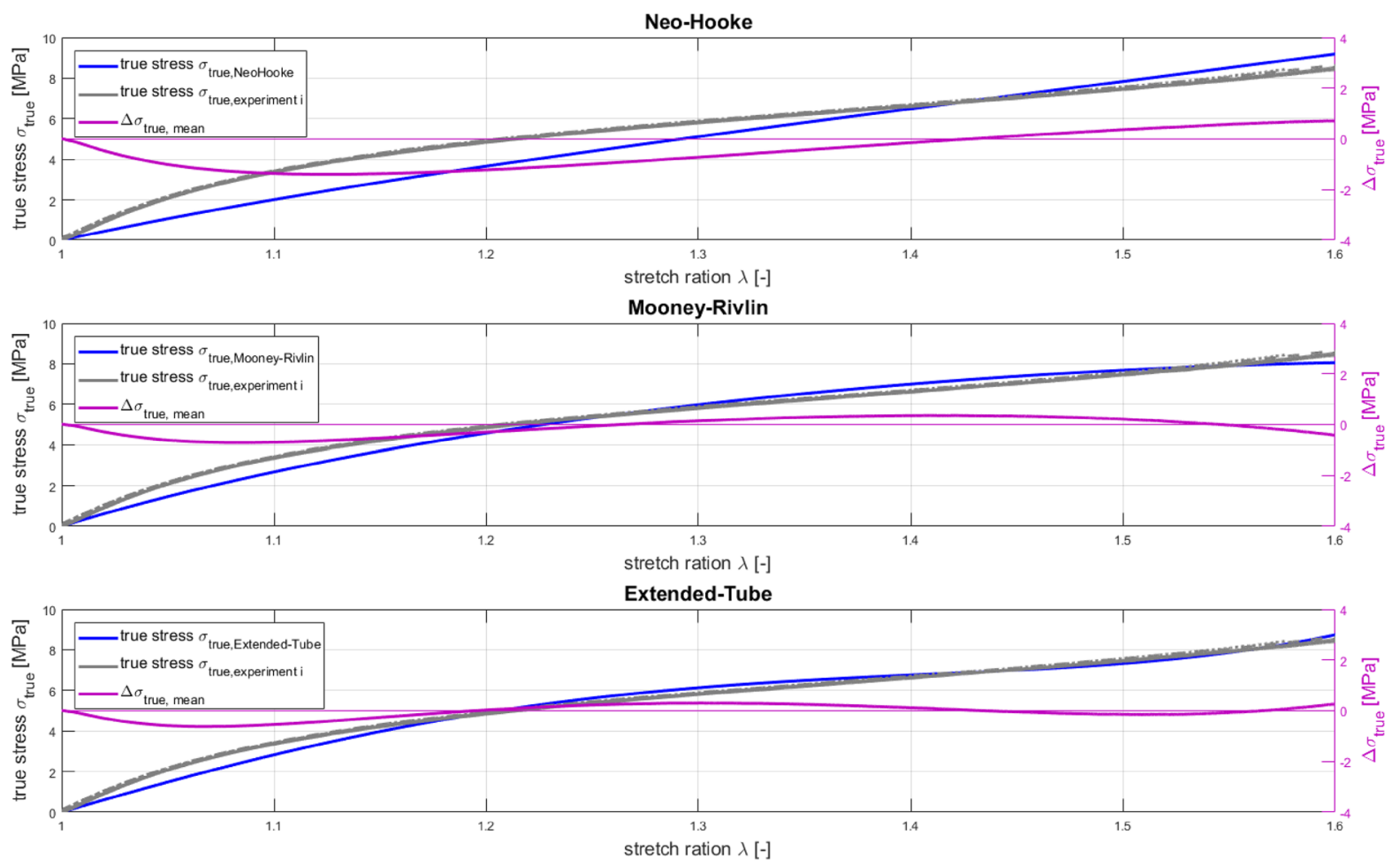

Fig. 27 Fitting different material models to the EVA sample data from the biaxial tensile test (BT)

able to approximate the curvatures for both test modes in a suitable way. When dividing the whole stretch axis into two regions of curvature (approx. at $\lambda=2.5$ ), one realizes, that for the UT as well as for the BT testing mode nor model captures the convex curvature (left part of the axis) very well, whereas for the right part of the stretch axis with the concave curvature the ET model is able to approximate the data well. Thus it can be concluded, that the ET model is able to approximate the real material behaviour in different testing 

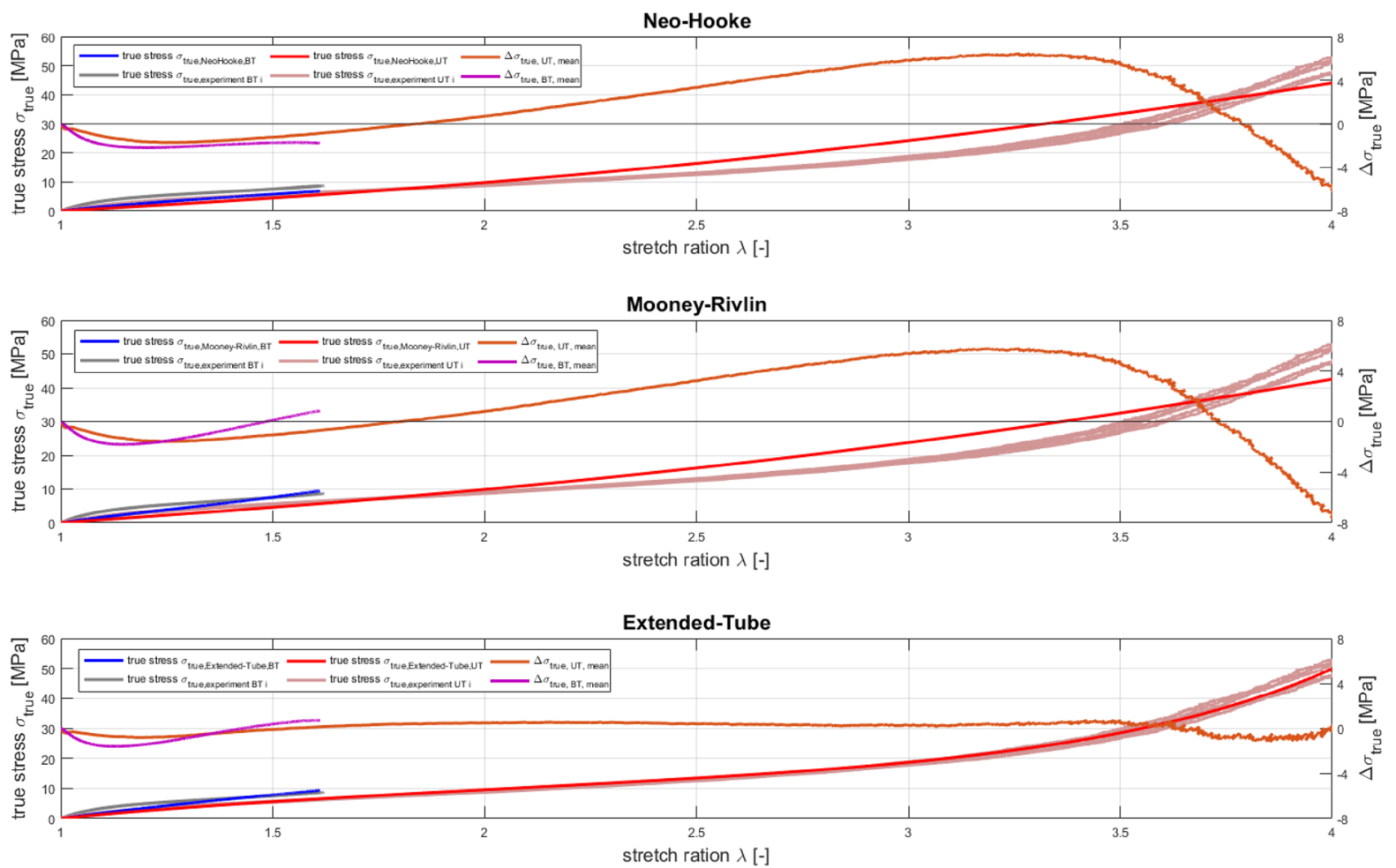

Fig. 28 Fitting different material models to the EVA sample data from the biaxial tensile test (BT) as well as the uniaxial tensile test (UT)

modes and is therefore in a simulation situation more accurately than the Neo-Hooke or the Mooney-Rivlin material model. Despite that, one has to keep in mind, that there is a strong dependency on the initial material parameter guess for the ET model. Furthermore, as already pointed out in Sect. 3.3, the three models capture the dependency of the Helmholz free energy on the two invariants of the left Cauchy-Green stretch tensor in different ways and are thus more or less able, to reproduce the response due to different testing scenarios from an energetic point of view.

\section{Discussion, conclusion und future research}

\subsection{Summary and discussion of the findings}

In this paper two aspects of the constitutive modelling of an EVA-based laminated safety glass interlayer were highlighted: the models and identification procedures for linear viscoelasticity as well as hyperelasticity.

Considering linear viscoelasticity, two different Prony-parameter identification methods based on exper- imental data have been introduced: a recently presented two-step data fit by Kuntsche (2015) and the novel method GUSTL by Kraus et al. The theoretical considerations were applied to test data in Sect. 4.1.3. It was found, that GUSTL is superior to existing methods when it comes to numerical effort. It should be noted here, that the Prony-parameter identification process was done with a Master Curve, which was created before the Prony-series identification with a TTSP on the experimental DMTA raw data. This Master Curve still needs to be validated in component tests, such as creep tests. Until now, only a horizontal shift is considered, which corresponds to relaxation times changes of the single Maxwell-Elements in the Generalized Maxwell-Model. This is a sufficient approximation for thermorheological simple materials. However, EVA is assigned to the group of elastomers, which show an increase in stiffness with temperature and hence indicate a thermorheological complex behaviour. Thus, further investigations on the TTSP have to be done for EVA.

Furthermore the fitting of hyperelastic material models against test data from uniaxial as well as biax- 
ial testing was investigated. Measurements in uniaxial and biaxial mode were used to calibrate nonlinear hyperelastic material models, where it was seen, that the ET model fits the data the best within the range of all tested material models in terms of the difference (between the mean value and the fitted value) in the true stress $\Delta \sigma_{\text {true. }}$. At this point the question on the necessity of testing further already existing Helmholz free energies or introducing a novel material model for EVA in terms of the Helmholz free energy can be raised. This is due to the fact, that two of the tree Helmholz free energy equations in the context of this paper do not take into account the second invariant $I_{2}$ of the left / right Cauchy-Green stretch tensor. Here it still has to be shown, that the determined parameters represent the performed experiments accurately in Finite-ElementSoftware-programs.

As shown by the experimental and theoretical investigations in this paper, a generalization of the model of linear viscoelasticity in terms of the Prony-series representation for the large strain case is necessary. In the context of this paper, solely material nonlinearity was considered (i.e. hyperelasticity), which corresponds to the situation of having large strains. In Marques and Creus (2012) and Brinson and Brinson (2015) further considerations on the handling of general types of nonlinearities can be found.

Starting from the small strain linear viscoelasticity equation using the stress relaxation function expressed as Prony-series, the hyperelastic shear stress function $\tau_{\text {hyper }}(\gamma)$ is integrated by parts to finally obtain [for further deduction c.f. Marques and Creus (2012), Brinson and Brinson (2015)] the expression given in Eq. (52).

$$
\begin{aligned}
\tau(t)= & \tau_{\text {hyper }}(\gamma(t)) \\
& -\int_{0}^{t} \dot{g}_{R}(t-s) \cdot \tau_{\text {hyper }}(\gamma(t)) d s
\end{aligned}
$$

This kind of ansatz will be considered in further works by the authors to develop models for a more realistic description of the post-breakage behaviour of laminated safety glass.

\subsection{Future research}

In the context of this paper only a few points of future research can be highlighted. The authors will concentrate on the following tasks:
- Reformulation of the Prony-parameter identification methods to incorporate uncertainties/measurement noise

- Reformulation of the Prony-parameter identification methods to incorporate thermorheological complex material behaviour

- Incorporation of hyperelasticity into the Generalized Maxwell Element

- Application of the 'large strain Generalized Maxwell Element' to investigate glass post-breakage

It is assumed, that the solution of the mentioned open research questions will advance the scientific as well as the engineering understanding of the phenomena of the behaviour of structural glass applications in the postbreakage state.

\section{References}

Barredo, J. et al.: Viscoelastic vibration damping identification methods. Application to laminated glass. Procedia Eng. 10, 3208-3213 (2011)

Becker, F.: Dissertation: Entwicklung einer Beschreibun gsmethodik für das mechanische Verhalten unverstärkter Thermoplaste bei hohen Deformationsgeschwindigkeiten. MLU Halle-Wittenberg, Halle-Wittenberg (2009)

Bennison, S. J. et al.: Laminated glass for blast mitigation: role of interlayer properties. In: Glass Processing Days 2005, Tempere, pp. 494-496 (2005)

Bridgestone Industrial Limited: Verbund-Sicherheitsglas mit einer Verbundfolie der Produktfamilie EVASAFE mit Ansatz eines Schubverbundes abz Z-70.3-197 (2014)

Brinson, H., Brinson, L.: Polymer Engineering Science and Viscoelasticity: An Introduction. Springer, Berlin, Heidelberg (2015)

DIN 18008: Glas im Bauwesen-Bemessungs- und Konstruktionsregeln (2010)

DIN EN ISO 527: Kunststoffe - Bestimmung der Zugeigenschaften (2012)

Domininghaus, H. et al:: Kunststoffe - Eigenschaften und Anwendungen - 7., neu bearbeitete und erweiterte Auflage. Berlin, Heidelberg: Springer (2008)

Drass, M., Schuster, M., Schneider, J.: Submitted to Proceedings IABSE: Comparison of Unconventional Testing Methods for Mechanical Characterization of Polymeric Materials in Modern Glass Structures. Vancouver (2017)

Du Pont de Nemours GmbH: Verbund-Sicherheitsglas aus SentryGlas SGP 5000 mit Schubverbund abz Z-70.3-170 (2011)

EN ISO 527: Plastics-determination of tensile properties (2012)

Ensslen, F.: Dissertation: Zum Tragverhalten von VerbundSicherheitsglas unter Berücksichtigung der Alterung der Polyvinylbutyral-Folie. Bochum (2005)

Göhler, J.: Dissertation: Das dreidimensionale viskoelastische Stoffverhalten im großen Temperatur- und Zeitbereicham beispiels eines in der automobilen Aufbau- 
und Verbindungstechnik verwendeten Epoxidharzklebstoffs. Dresden (2010)

Grellmann, W., Seidler, S.: Kunststoffprüfung. Carl Hanser Verlag, München (2011)

Hooper, P.: Blast performance of silicone-bonded laminated glass. Springer, London (2011)

Kaliske, M., Heinrich, G.: An extended tube-model for rubber elasticity: statistical-mechanical theory and finite element implementation. Rubber Chem. Technol. 72(4), 602-632 (1999)

Kasper, R.: Dissertation: Tragverhalten von Glasträgern. Shaker Verlag, Aachen (2005)

Keuerleber, M.: Dissertation: Bestimmung des Elastizitätsmoduls von Kunststoffen bei hohen Dehnraten am Beispiel von PP. Stuttgart (2006)

Kothe, M.: Dissertation: Alterungsverhalten von piolymeren Zwischenschichtmaterialien im Bauwesen. Dresden (2013)

Kraus, M.A., Niederwald, M.: Rheological modelling of linear viscoelastic materials for strengthening measures in bridge engineering. In: Proceedings of the 11th German Japanese Bridge Symposium. OIT Osaka (2016)

Kraus, M.A., Niederwald, M.: Generalized collocation method using Stiffness matrices in the context of the Theory of Linear viscoelasticity (GUSTL). Tech. Mech (submitted)

Kuntsche, J.K. et al.: Conference Proceedings GPD: Viscoelastic Properties of Laminated Glass Interlayers-Theory and Experiments. Tampere (2015)

Kuntsche, J.K.: Dissertation: Mechanisches Verhalten von Verbundglas unter zeitabhängiger Belastung und Explosionsbeanspruchung. Darmstadt (2015)
Kuraray Europe GmbH Division TROSIFOL: VerbundSicherheitsglas mit der PVB-Folie TROSIFOL ES mit Schubverbund abz Z-70.3-236 (2014)

Marques, S., Creus, G.: Computational Viscoelasticity. Springer, Berlin (2012)

NEN 2608: (nl) Vlakglas voor gebouwen - Eisen en bepalingsmethode (2014)

Sackmann, V.: Dissertation: Untersuchungen zur Dauerhaftigkeit des Schubverbundes von Verbundsicherheitsglas mit verschiedenen Folien aus Polyvinylbutyral. München (2008)

Schneider, J., Kolling, S., Kuntsche, J.M., Monnich, S.: Proceedings of Engineered Transparency: Tensile Properties of Different Polymer Interlayers Under Highstrain Rates. Düsseldorf (2012)

Schuler, C.: Dissertation: Einfluss des Materialverhaltens von Polyvinylbutyral auf das Tragverhalten von Verbundsicherheitsglas in Abhängigkeit von Temperatur und Belastung. München (2003)

Schwarzl, F.: Rudolf Polymermechanik. Springer, Berlin (1990)

Stommel, M., Stojek, M., Korte, W.: FEM zur Berechnung von Kunststoff- und Elastomerbauteilen. Carl Hanser Verlag, Munich (2011)

Tschoegl, N.: The Phenomenological Theory of Linear Viscoelastic Behavior-An Introduction. Springer, Berlin (1989) 